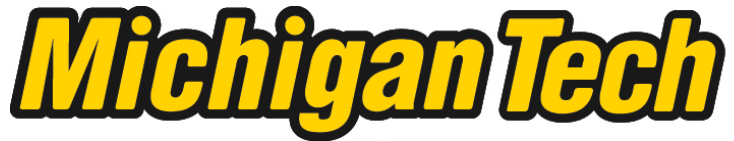 \\ Michigan Technological University Create the Future Digital Commons @ Michigan Tech
}

\section{Pulse polarization radar for determining spatial and material information about a target in orbit}

Nicholas D. Peterson

Michigan Technological University

Follow this and additional works at: https://digitalcommons.mtu.edu/etds

Part of the Electrical and Computer Engineering Commons

Copyright 2009 Nicholas D. Peterson

\section{Recommended Citation}

Peterson, Nicholas D., "Pulse polarization radar for determining spatial and material information about a target in orbit", Master's Thesis, Michigan Technological University, 2009.

https://doi.org/10.37099/mtu.dc.etds/44

Follow this and additional works at: https://digitalcommons.mtu.edu/etds

Part of the Electrical and Computer Engineering Commons 


\title{
Pulse Polarization Radar for Determining Spatial and Material Information about a Target in Orbit
}

\author{
Nicholas D. Peterson
}

A thesis submitted in partial fulfillment of the requirements for the degree of Master of Science in Electrical Engineering

Michigan Technological University

2009

Copyright $($ ) Nicholas D. Peterson 2009 
This thesis, "Pulse Polarization Radar for Determining Spatial and Material Information about a Target in Orbit" is hereby approved in partial fulfillment of the requirements for the Degree of MASTER OF SCIENCE IN ELECTRICAL ENGINEERING.

Department: Electrical and Computer Engineering

Thesis Advisor:

Michael C. Roggemann

Department Chair:

Warren Perger

Date: 


\section{Acknowledgments}

I would like to thank the following people for helping me get as far as I have today. First of all I would like to thank my advisor Mike Roggemann. Not only did you give me this chance to continue my education on the graduate level, but you have helped me at every step along the way. I would also like to thank Dan Hand and John Stryjewski from CSC and Dave Tyler from the University of Arizona. John, thank you for developing the Microfacet scattering model that I used in this thesis. Dan, without your help I would have never figured out Linux. Dave, thank you for your help, especially in figuring out how to make the SNR measurements. A few of my undergraduate and high school professors also deserve a large thank you for starting me off on this path. Rob Jacobs, who introduced me to both electronics and the dead skunk song. Jim Lund, for giving me my start in Physics. Chuck Niederriter, for your ability to make any physics example fun with beer trucks and falling monkeys. And finally my undergraduate advisor Dennis Henry for giving me the motivation and confidence to enter grad school. I hope your train set is twice as big now. 


\section{Contents}

Acknowledgments

1 Introduction 1

2 Background $\quad 3$

2.1 Scalar Diffraction Theory . . . . . . . . . . . . . . . 3

2.2 The Fresnel Approximation . . . . . . . . . . . . . . . . . 4

2.3 The Fraunhofer Approximation . . . . . . . . . . . . . . . . 6

2.4 Surface Scattering Basics . . . . . . . . . . . . . . . . . . 6

2.5 Bidirectional Reflective Distribution Function . . . . . . . . . . . . . 8

2.6 Rayleigh-Rice Vector Perturbation Theory . . . . . . . . . . . . . . 8

2.7 Beckmann-Kirchhoff Surface Scatter Theory . . . . . . . . . . . . . . 10

2.8 Microfacet Scattering Model . . . . . . . . . . . . . . . . . . . . . 13

2.9 Modeling the Atmosphere . . . . . . . . . . . . . . . . . 16

2.10 Propagation Model . . . . . . . . . . . . . . . . . . . 20

2.11 SNR of the Atmospheric Model . . . . . . . . . . . . 23

3 Construction of System Model 26

3.1 Target Creation . . . . . . . . . . . . . . . . 26

3.2 Implementation of the Rayleigh-Rice Model . . . . . . . . . . . . . . . . . 32

3.3 Implementation of Beckmann-Kirchhoff Model . . . . . . . . . . . . . . . . 32

3.4 Implementation of Microfacet Scattering Model . . . . . . . . . . . . . 35

4 Results 39

4.1 Backscattering from Rayleigh-Rice Surface . . . . . . . . . . . . . . . . 39

4.2 Backscattering from Beckmann-Kirchhoff Surface . . . . . . . . . . . . . 40

4.3 Backscattering from Microfacet Surface . . . . . . . . . . . . . . . 48

5 Conclusion $\quad \mathbf{5 1}$

5.1 Discussion of Results . . . . . . . . . . . . . . . . 51 
5.2 Areas for Future Work . . . . . . . . . . . . . . . . 52

Bibliography 


\section{List of Figures}

2.1 Geometry for Huygens-Fresnel Principle . . . . . . . . . . . . . . . . 4

2.2 Light scattered by an imperfect surface . . . . . . . . . . . . . . . . 7

2.3 The surface profile and its corresponding statistical parameters[3] $\ldots \ldots$. . 7

2.4 Relationship between surface profile and surface PSD functions[3] . . . . . 7

2.5 Geometry for the calculation of the BRDF . . . . . . . . . . . 8

2.6 Geometry for Rayleigh-Rice Theory defining $s$ and $p$ polarization components[3] 9

2.7 Example microfacet surface. The blue lines represent the surface normals of each microfacet.[15] . . . . . . . . . . . . . . . . . . . 14

2.8 Side view of surface composed of microfacets. The green region is illuminated from direction $\vec{k}$ and the gray region is not illuminated, resulting in obscured microfacets. . . . . . . . . . . . . . . . . 15

2.9 Structure function as a function of altitude for the night sky of Maui, Hawaii 18

2.10 Layer atmospheric seeing as a function of altitude for the night sky of Maui, Hawaii . . . . . . . . . . . . . . . . . . 18

2.11 The 2-D cusp-like arrays for the $x$ and $y$ direction respectively. . . . . . . . 19

$2.12 R^{2}(x, y)$, the sum of the two cusp arrays. . . . . . . . . . . . . . 19

2.13 Example phase screens with $n=350, l=14 \mathrm{~m}$ and $r_{0}=.4144 \mathrm{~cm} \ldots \ldots . .20$

2.14 System model illustrating atmosphere and target in orbit (Not to scale) . . . 21

2.15 Four images of the absolute value of the optical field propagating through the 10 phase screen atmospheric model. Top Left: Field exiting transmitter. Top Right: Field after 2 phase screens. Lower Left: Field after 6 phase screens. Lower Right: Field after 10 phase screens. This is the field that will be sent to the target. . . . . . . . . . . . . . . . 22

2.16 Example virtual aperture surrounding the optical field on axis. Using the statistics of the energy captured by the aperture, the SNR of the system can be found. . . . . . . . . . . . . . . . 23

2.17 Logarithm of the SNR as a function of propagation distance for various aperture diameters. Values found from 1000 separate atmospheric iterations with a transmitter diameter of $.6 \mathrm{~m}$. 
2.18 SNR as a function of aperture diameter in the target plane. Values found from 2000 separate atmospheric iterations with a transmitter diameter of .6

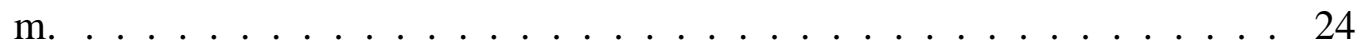

2.19 SNR as a function of aperture diameter in the target plane. Values found from 1000 separate atmospheric iterations with transmitter diameter of $.6 \mathrm{~m} . \quad 25$

3.1 Example depth map for a conical target with coordinate description. $Z$-axis is positive out of the page. Transmitter/receiver setup is along the $z$-axis. . . 27

3.2 Angle found from depth map in figure 3.1. Left: Angle between normal vector and z-axis $\left(\theta_{i}\right)$ in radians. Right: Angle between normal vector and y-axis $(\gamma)$ in radians. . . . . . . . . . . . . . . 27

3.3 Geometry for conversion between $x-y$ polarization and $s-p$ polarization coordinates on conical target pointing along positive $z$-axis. . . . . . . . . 28

3.4 Cross-section of target. The red arrows indicate incoming and scattered radiation of interest. The black arrow shows the surface normal, from which both $\theta_{i}$ and $\theta_{s}$ are measured. . . . . . . . . . . . . . . . . . 29

3.5 Consecutive slices of a conical target oriented pointing downward. Red values are equal to one, while blue values are equal to zero. . . . . . . . . . 29

3.6 Left: Two entire Hanning slices. Right: Close up of overlapping region. At every horizontal location the sum of the two edges is equal to unity. . . . . 30

3.7 Consecutive slices of a conical target oriented pointing downward utilizing Hanning edges. . . . . . . . . . . . . . . . . 30

3.8 Radiation scattered back to the aperture plane from identical slices of a conical target pointing towards the top right corner of the array. The left image was constructed using the original slice forming algorithm and shows evidence of ringing. The right image was formed using slices with Hanning

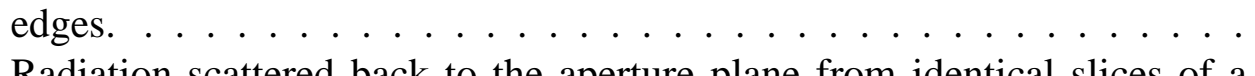

3.9 Radiation scattered back to the aperture plane from identical slices of a conical target pointing upward. The left image was constructed using the original slice forming algorithm and shows evidence of ringing. The right image was formed using slices with Hanning edges. . . . . . . . . . . . . . 31

3.10 Scattering distributions using R-R model with $\sigma_{s} / \lambda=.02$ and an incident angle of $0^{\circ} \ldots \ldots \ldots \ldots \ldots \ldots$

3.11 Scattering distributions using R-R model with $\sigma_{s} / \lambda=.01$ and an incident angle of $0^{\circ} \ldots \ldots \ldots \ldots \ldots \ldots$

3.12 Scattering distributions using R-R model with $\sigma_{s} / \lambda=.02$ and an incident

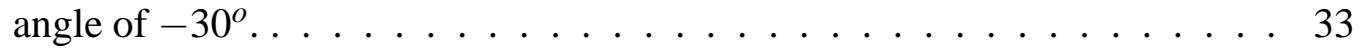

3.13 Scattering distributions using R-R model with $\sigma_{s} / \lambda=.01$ and an incident angle of $-30^{\circ} \ldots \ldots \ldots \ldots \ldots \ldots$. . . . . . . . . . . 34 
3.14 Sum in equation 3.20 as a function of maximum limit of $m$ showing convergence. In the left figure, $l_{c} / \lambda=2$ and $\theta_{i}=\theta_{s}=0^{\circ}$. In the right figure, $l_{c} / \lambda=2$ and $\theta_{i}=\theta_{s}=40^{\circ} \ldots \ldots \ldots \ldots \ldots$

3.15 Scattering distributions using B-K model with $\sigma_{s} / \lambda=.25$ and an incident angle of $0^{\circ} \ldots \ldots \ldots \ldots \ldots$

3.16 Scattering distributions using B-K model with $\sigma_{s} / \lambda=.1$ and an incident

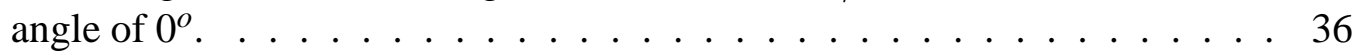

3.17 Scattering distributions using B-K model with $\sigma_{s} / \lambda=.25$ and an incident angle of $30^{\circ} \ldots \ldots \ldots \ldots \ldots$. . . . . . . . . . . . . . . . . . . . . . . .

3.18 Scattering distributions using B-K model with $\sigma_{S} / \lambda=.1$ and an incident angle of $30^{\circ} \ldots \ldots \ldots \ldots \ldots$. . . . . . . . . . . . . . . . . . . . .

4.1 Returning $x$-polarized fields at receiver plane for R-R scattering model, where $\sigma_{s} / \lambda=.02$ and $l_{c} / \lambda=2 \ldots \ldots \ldots \ldots$. . . . . . . . 40

4.2 Returning $y$-polarized fields at receiver plane for R-R scattering model, where $\sigma_{s} / \lambda=.02$ and $l_{c} / \lambda=2 \ldots \ldots \ldots$. . . . . . . . . 41

4.3 Returning $x$-polarized fields at receiver plane for R-R scattering model, where $\sigma_{s} / \lambda=.02$ and $l_{c} / \lambda=2 \ldots \ldots \ldots$. . . . . . . . . 41

4.4 Returning $y$-polarized fields at receiver plane for R-R scattering model, where $\sigma_{s} / \lambda=.02$ and $l_{c} / \lambda=2 \ldots \ldots \ldots$. . . . . . . . . 41

4.5 Returning $x$-polarized fields at receiver plane for R-R scattering model, where $\sigma_{s} / \lambda=.02$ and $l_{c} / \lambda=2 \ldots \ldots \ldots \ldots$. . . . . . . . 42

4.6 Returning $y$-polarized fields at receiver plane for R-R scattering model, where $\sigma_{s} / \lambda=.02$ and $l_{c} / \lambda=2 \ldots \ldots \ldots$. . . . . . . . . 42

4.7 Energy of $x$-polarized pulse in aperture plane as a function of time of arrival and surface roughness for the R-R scattering model. The target is the downward pointing cone. $l_{c} / \lambda$ is held constant at $2 \ldots \ldots \ldots \ldots$

4.8 Energy of $y$-polarized pulse in aperture plane as a function of time of arrival and surface roughness for the R-R scattering model. The target is the downward pointing cone. $l_{c} / \lambda$ is held constant at $2 . . . . . . .$.

4.9 Energy of $x$-polarized pulse in aperture plane as a function of time of arrival and surface roughness for the R-R scattering model. The target is the angled cone. $l_{c} / \lambda$ is held constant at $2 \ldots \ldots \ldots \ldots$

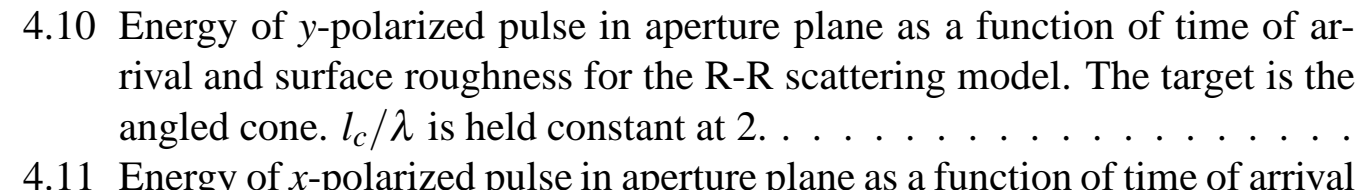

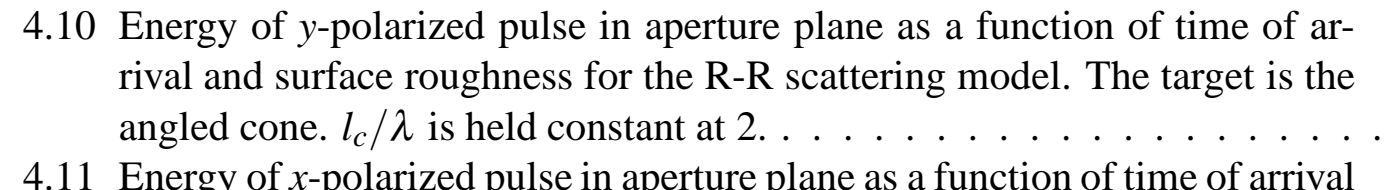
and autocovariance length for the R-R scattering model. The target is the downward pointing cone. $\sigma_{s} / \lambda$ is held constant at .02 . 
4.12 Energy of $y$-polarized pulse in aperture plane as a function of time of arrival and autocovariance length for the R-R scattering model. The target is the

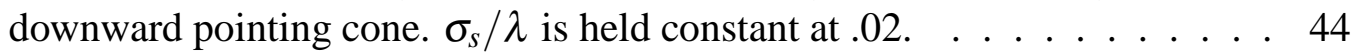

4.13 Energy of $x$-polarized pulse in aperture plane as a function of time of arrival and autocovariance length for the R-R scattering model. The target is the

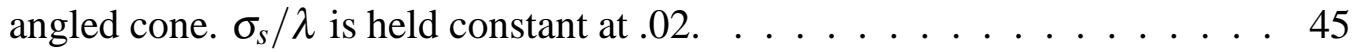

4.14 Energy of $y$-polarized pulse in aperture plane as a function of time of arrival and autocovariance length for the R-R scattering model. The target is the angled cone. $\sigma_{s} / \lambda$ is held constant at $.02 \ldots . . . . . . . . . . .45$

4.15 Returning fields at receiver plane for B-K scattering model, where $\sigma_{s} / \lambda=$ .1 and $l_{c} / \lambda=2 \ldots \ldots \ldots \ldots \ldots \ldots \ldots$

4.16 Returning fields at receiver plane for B-K scattering model, where $\sigma_{s} / \lambda=$ .5 and $l_{c} / \lambda=2 \ldots \ldots \ldots \ldots$. . . . . . . . . . . . . . . . . . 47

4.17 Returning fields at receiver plane for B-K scattering model, where $\sigma_{s} / \lambda=$

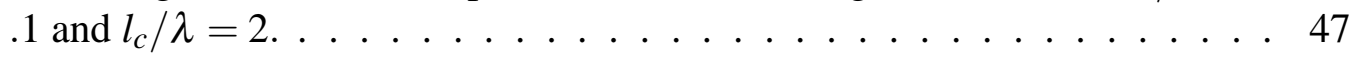

4.18 Energy of pulse in aperture plane as a function of time of arrival and autocovariance length for the B-K scattering model. The target is the downward pointing cone. $\sigma_{s} / \lambda$ is held constant at $.25 \ldots \ldots . \ldots . . \ldots 48$

4.19 Energy of pulse in aperture plane as a function of time of arrival and surface roughness for the B-K scattering model. The target is the downward pointing cone. $l_{c} / \lambda$ is held constant at $\ldots \ldots$. . . . . . . . . . . 48

4.20 Returning $x$-polarized fields at receiver plane for MF scattering model. . . . 49

4.21 Returning $y$-polarized fields at receiver plane for MF scattering model. . . . 49

4.22 Returning $x$-polarized fields at receiver plane for MF scattering model. . . . 50

4.23 Returning $y$-polarized fields at receiver plane for MF scattering model. . . . 50

5.1 Horizontal slice across center of return pulse exhibiting sinc function behavior. . . . . . . . . . . . . . . . . . 52 


\section{Chapter 1}

\section{Introduction}

Traditional imaging systems can be used to determine the orientation and shape of satellites that are large or are in low orbits[15]. Difficulty arises when trying to image satellites which are smaller and/or in higher orbits. Traditional imaging systems also rely on capturing reflected sunlight, so passive systems are limited to certain time-windows. Ideally, passive measurements are made when the satellite is illuminated by the sun, but the observatory is not. Using an intensity based approach encounters difficulties with atmospheric extinction, whether the system is passive or utilizes active laser illumination. The atmosphere also limits the angular resolution as the receiver. A viable technique must utilize active illumination as well as provide spacial and material information about a object in orbit.

Pulse Polarization Radar (PPR) uses very short laser pulses, on the order of a few picoseconds, which are reflected off an object in orbit. An elongation of the return pulse will occur due to the physical depth of the object. The polarization of the return pulse, as a function of time, can be measured to provide the necessary spatial and material properties of the object. Fortunately, the atmosphere does not change the polarization of a pulse during propagation. The polarization of the pulse is however altered by scattering off the object.

A model of the atmosphere will be constructed using Kolmogorov phase screens. Fresnel diffraction is used for free space propagation of the laser pulse. Three different scattering models are introduced to simulated scattering from the target object for different surface characteristics. Rayleigh-Rice Perturbation theory[3, 4] is used to handle scattering from objects with surface features much smaller than the illuminating wavelength. When the surface features are on the order of the illuminating wavelength, the Beckmann-Kirchhoff scattering model [3] is used. A variation on the microfacet model of Ashikhmin et al [15] is used when the surface features are much larger than the illuminating wavelength. By observing the time history of the returning optical field at the receiver, information about the shape and orientation of the target is obtained. The total energy of the return pulse as a function of time provides information about the material used to construct the target. When 
the surface properties of the target are adjusted, the returning energy varies. This relationship can be used to create a look-up table for various satellite construction materials.

The remainder of this thesis is organized as follows. Chapter two provides background information on diffraction theory and modeling atmospheric turbulence, introduces the three scattering models and constructs the propagation model. Chapter three uses knowledge of the surface features, such as the surface power spectral density (PSD) and the autocovariance function (ACV), to implement the scattering models to specific surfaces. Chapter four describes the backscattering from the target object and gives the results gained by utilizing the specific scattering models. Chapter five summarizes the results and suggests areas for future work. 


\section{Chapter 2}

\section{Background}

\subsection{Scalar Diffraction Theory}

Arnold Sommerfeld defined diffraction as "any deviation of light rays from rectilinear paths which cannot be interpreted as reflection or refraction"[1]. Diffraction arises from the spatial confinement of a wavefront. The first accurate description of diffraction was published in 1665 by Francesco Grimaldi, who called the phenomenon "diffractio"[2]. His book, De Lumine, included a description of the bands of light formed when a thin rod was illuminated by a small source [20].

Robert Hooke included a description of colored interference patterns generated by thin films in his 1665 book Micrographia[18]. By illuminating a bird feather with sunlight, James Gregory observed a central white spot surrounded by colored components of the sunlight[3]. In 1690, Christiaan Huygens published Traité de la Lumière[10], which included what is now called Huygens' Principle. Huygens' Principle states that every point on a propagating wavefront serves as the source of spherical secondary wavelets, such that the wavefront at some later time is the envelope of these wavelets[2, 10]. The laws of refraction and reflection can be derived from this principle[3]. In his 1704 work, Opticks, Issac Newton strongly supported the corpuscular theory of light over the wave theory [17]. Because of Newton's enormous prestige, little work was done on the wave theory for much of the 18th century[1]. In 1802 and 1803, Thomas Young presented papers to the Royal Society in London supporting the wave theory of light and introducing the controversial idea of interference [19]. By allowing Huygens' secondary wavelets to mutually interfere, Augustin Jean Fresnel was able to introduce a wave theory which accounted for reflection, refraction, and diffraction. Fresnel presented his theory to a prize committee of the French Academy of Sciences. This theory was initially ridiculed by the judges, including Siméon Poisson, because it predicted a bright spot in the shadow cast by an opaque disk. When the experiment was preformed however, the predicted bright spot was found and dubbed 


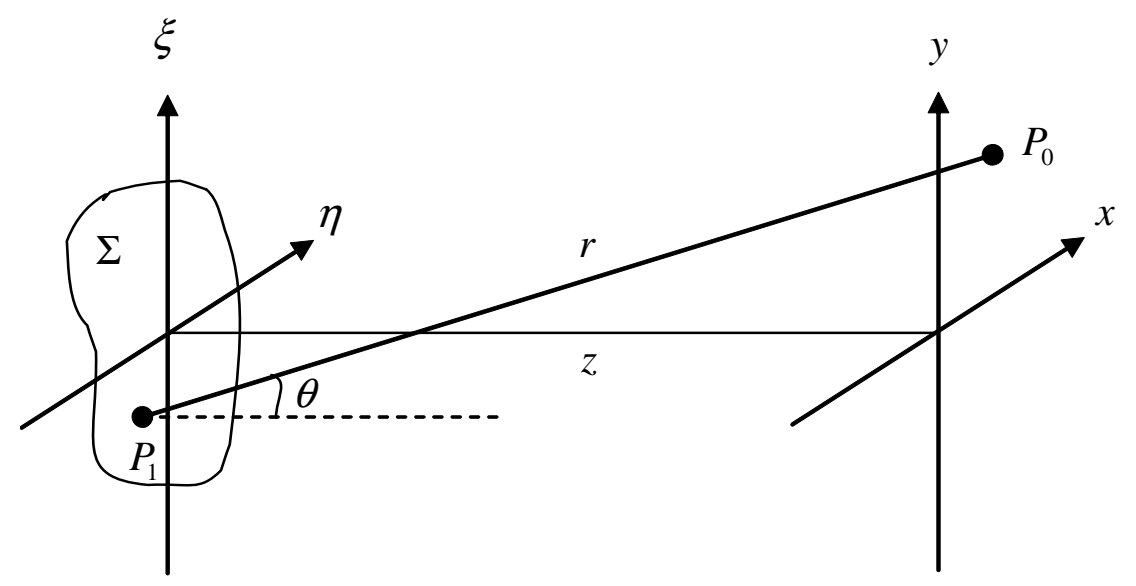

Figure 2.1: Geometry for Huygens-Fresnel Principle

"Poisson's Spot" [1].

If each point in a diffracting aperture is treated as a source of spherical waves, the superposition of these waves produces the Huygens-Fresnel Principle

$$
U(x, y)=\frac{1}{j \lambda} \int_{-\infty}^{\infty} \int_{-\infty}^{\infty} U_{0}(\xi, \eta) \frac{\exp (j k r)}{r} \cos \theta \mathrm{d} \xi \mathrm{d} \eta,
$$

where $\Sigma$ is the extent of the diffraction aperture in the $(\xi, \eta)$ plane and $U_{0}(\xi, \eta)$ is the optical disturbance exiting the aperture. The distance $r$ is given exactly by

$$
r=\sqrt{z^{2}+(x-\xi)^{2}+(y-\eta)^{2}} .
$$

\subsection{The Fresnel Approximation}

The Huygens-Fresnel Principle in equation 2.1 can be reduced to simpler and more useful forms by making approximations on the value of $r$. For the Fresnel approximation, $r$ is replaced by $z$ in the denominator of equation 2.1. In the exponential $r$ is multiplied by the large number $k$, so small approximation errors become much larger. In this case, $r$ is substituted by the first two terms of the binomial expansion of equation 2.2, such that

$$
r \approx z\left[1+\frac{1}{2}\left(\frac{x-\xi}{z}\right)^{2}+\frac{1}{2}\left(\frac{y-\eta}{2}\right)^{2}\right] .
$$


This assumption will be valid, as long as the following Fresnel condition is met[1]:

$$
z^{3} \gg \frac{\pi}{4 \lambda}\left[(x-\xi)^{2}+(y-\eta)^{2}\right]_{\max }^{2}
$$

Using the Fresnel approximation, equation 2.1 can be rewritten [3]

$$
U(x, y)=\frac{\exp (j \lambda z)}{j \lambda} \int_{-\infty}^{\infty} \int_{-\infty}^{\infty} U_{0}(\xi, \eta) \exp \left\{\frac{j k}{2 z}\left[(x-\xi)^{2}+(y-\eta)^{2}\right]\right\} \mathrm{d} \xi \mathrm{d} \eta
$$

Equation 2.5 can be rewritten as a convolution integral in the form [1]

$$
U(x, y)=\int_{-\infty}^{\infty} \int_{-\infty}^{\infty} U_{0}(\xi, \eta) h(x-\xi, y-\eta) \mathrm{d} \xi \mathrm{d} \eta
$$

where the convolution kernel is

$$
h(x, y)=\frac{\exp (j k z)}{j \lambda z} \exp \left[\frac{j k}{2 z}\left(x^{2}+y^{2}\right)\right] .
$$

Another useful form of equation 2.5 can be found by factoring the term [1]

$$
\exp \left[\frac{j k}{2 z}\left(x^{2}+y^{2}\right)\right]
$$

outside of the integral, resulting in the form of a Fourier transform integral

$$
U(x, y)=\frac{\exp (j k z)}{j \lambda z} \exp \left[\frac{j k}{2 z}\left(x^{2}+y^{2}\right)\right] \int_{-\infty}^{\infty} \int_{-\infty}^{\infty} K(x, y, \xi, \eta) \mathrm{d} \xi \mathrm{d} \eta .
$$

where $K(x, y, \xi, \eta)$ is given by

$$
K(x, y, \xi, \eta)=U(\xi, \eta) \exp \left[\frac{j k}{2 z}\left(\xi^{2}+\eta^{2}\right)\right] \exp \left[-\frac{2 \pi j}{\lambda z}(x \xi+y \eta)\right]
$$




\subsection{The Fraunhofer Approximation}

By applying the more stringent Fraunhofer condition [1]

$$
z \gg \frac{k\left(\xi^{2}+\eta^{2}\right) \max }{2}
$$

further simplification can be made to equation 2.9. Under this condition, the quadratic phase term in equation 2.9 evaluates to approximately unity over the diffracting aperture [3]

$$
U(x, y)=\frac{\exp (j k z)}{j \lambda z} \exp \left[\frac{j k}{2 z}\left(x^{2}+y^{2}\right)\right] \int_{-\infty}^{\infty} \int_{-\infty}^{\infty} U_{0}(\xi, \eta) \exp \left[\frac{-2 \pi j}{h \lambda}(x \xi+y \eta)\right] \mathrm{d} \xi \mathrm{d} \eta
$$

Aside from the factors outside of the integrand, equation 2.12 is simply the Fourier transform of the optical disturbance just after the aperture plane evaluated at [1]

$$
f_{x}=\frac{x}{\lambda z}
$$

and

$$
f_{y}=\frac{y}{\lambda z}
$$

\subsection{Surface Scattering Basics}

When light strikes any real surface, the reflected radiation consists of both specularly and diffusely reflected components as shown in figure 2.2[3]. A surface is described by the surface profile. This is a two dimensional function $h(x, y)$ which give the height of the surface at each point. Besides the surface profile itself, scattered light is governed by the surface height distribution function and the surface ACV, both of which are statistical in nature. The relationship between these three functions is shown in figure 2.3 [3].

In many cases of interest, the surface height distribution function can be modeled as Gaussian. When this is the case, the standard deviation is called the root-mean-square (RMS) surface roughness or $\sigma_{s}$. The half-width at $1 / e$ height of the surface ACV function is called the autocovariance length, $l_{c}$. Unlike the surface height distribution function, the surface ACV function is not necessarily assumed to be Gaussian.

Another important surface parameter is the surface power spectral density (PSD) function. The surface PSD function and surface ACV function form a Fourier transform pair, as illustrated in figure $2.4[3,8]$. 


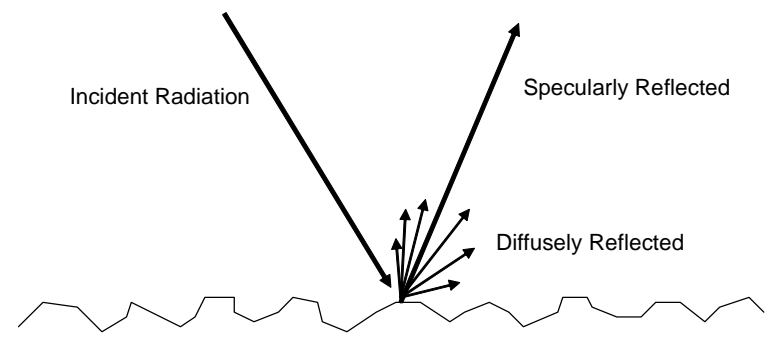

Figure 2.2: Light scattered by an imperfect surface

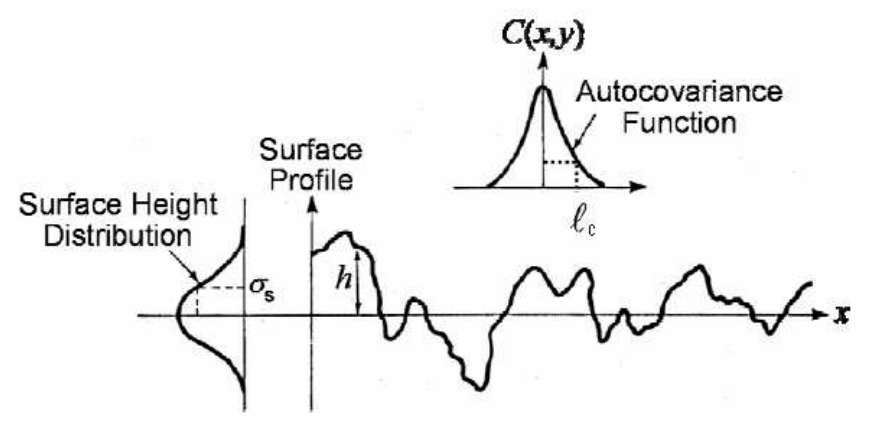

Figure 2.3: The surface profile and its corresponding statistical parameters[3]
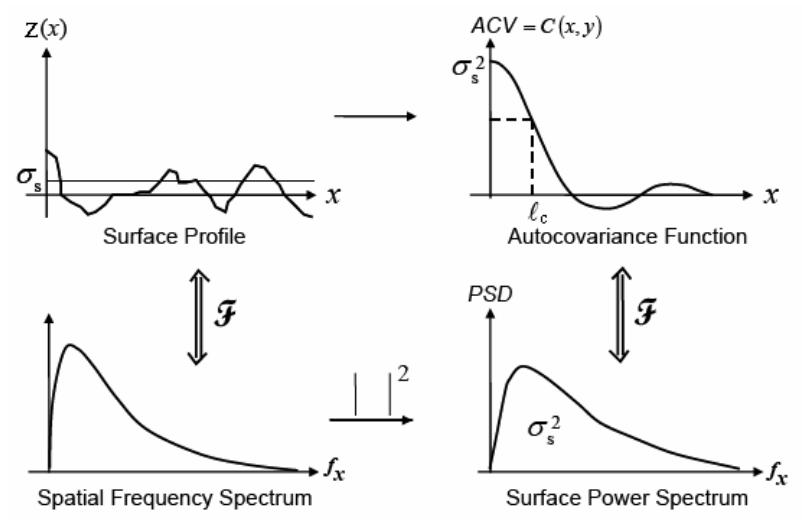

Figure 2.4: Relationship between surface profile and surface PSD functions[3] 


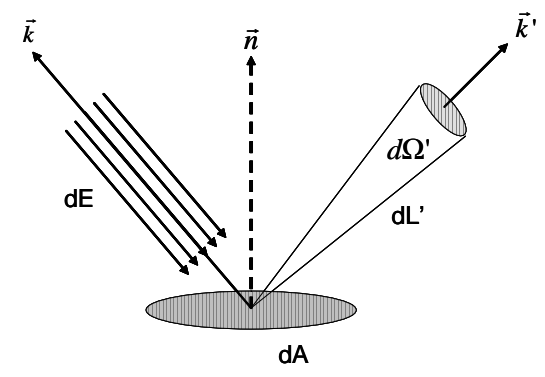

Figure 2.5: Geometry for the calculation of the BRDF

\subsection{Bidirectional Reflective Distribution Function}

In 1970, F. E. Nicodemus introduced the bidirectional reflective distribution function to quantitatively describe scattering from an imperfect surface[4]. It is defined as the radiance reflected by a surface differential into a differential solid angle divided by the incident irradiance[3].

$$
B R D F \equiv \frac{\mathrm{d} L_{s}}{\mathrm{~d} E_{i}} \simeq \frac{\mathrm{d} P_{s} / \mathrm{d} \Omega_{s} \mathrm{~d} A \cos \theta_{s}}{P_{i} / \mathrm{d} A} \simeq \frac{P_{s} / \Omega_{s}}{P_{i} \cos \theta_{s}}
$$

Here irradiance is given in terms of light flux per unit surface area (not beam cross section). Radiance has units of flux per unit surface area per unit solid angle, and irradiance has units of flux per unit area [4]. This gives the BRDF the units of $1 / s r$. The cosine term is a factor used to correct the size of the differential area, $\mathrm{d} A$, to its apparent size when viewed from the direction of the scatter. The geometry for the calculation of the BRDF is given in figure 2.5 where $\mathrm{d} E$ is the incident irradiance and the $\mathrm{d} L$ ' is the scattered radiance [3].

\subsection{Rayleigh-Rice Vector Perturbation Theory}

When surface roughness is small compared to the wavelength of incident radiation $\left(\sigma_{s} \ll \lambda\right.$ ), the Rayleigh-Rice Perturbation theory (R-R) can be used to model scattering [3,4]. This theory treats surface elements as small deviations from a perfectly smooth surface. The derivation of this theory is beyond the scope of this chapter, but its result will be used later. This theory relates the intensity scattered through the solid angle $\mathrm{d} \Omega_{s}$ per unit incident power to the PSD of the scattering surface

$$
\frac{I_{s}}{P_{i}}=\frac{\left(\mathrm{d} P_{s} / \mathrm{d} \Omega_{s}\right)}{P_{i}}=\left(\frac{16 \pi^{2}}{\lambda^{4}}\right) Q \cos \theta_{i} \cos ^{2} \theta_{s} \operatorname{PSD}\left(f_{x}, f_{y}\right) .
$$

$Q$ is a real, dimensionless polarization dependent reflectance term, and $\operatorname{PSD}\left(f_{x}, f_{y}\right)$ represents the surface power spectral density evaluated at 


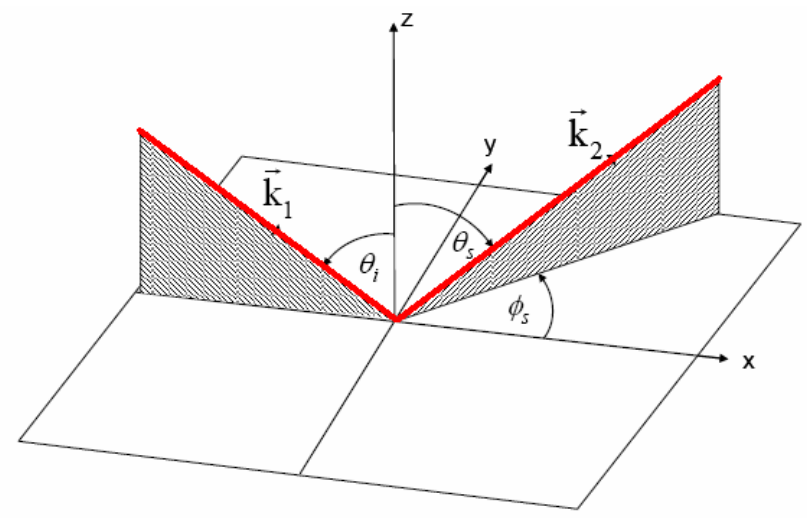

Figure 2.6: Geometry for Rayleigh-Rice Theory defining $s$ and $p$ polarization components[3]

$$
f_{x}=\frac{\sin \theta_{s} \cos \varphi_{s}-\sin \theta_{i}}{\lambda}
$$

and

$$
f_{y}=\frac{\sin \theta_{s} \sin \varphi_{s}}{\lambda}
$$

The value of $Q$ depends on the surface orientation and dielectric constant of the object material, $\varepsilon$, as well as the incident polarization and the polarization discrimination of the detector. The polarization states are defined on the sample surface shown in figure 2.6 [3]. Notice that the $p$ components are parallel to the plane of propagation, while the $s$ components are perpendicular. Also, the direction of incidence is always in the $x z$ plane so $\varphi_{i}$ will always be equal to zero.

$Q$ can include up to 4 different components depending on the incident and observed polarization. For a $s$-polarized source and a sensor which detects only s-polarized light

$$
Q=Q_{s s}=\left|\frac{(\varepsilon-1) \cos \varphi_{s}}{\left(\cos \theta_{i}+\sqrt{\varepsilon-\sin ^{2} \theta_{i}}\right)\left(\cos \theta_{s}+\sqrt{\varepsilon-\sin ^{2} \theta_{s}}\right)}\right|^{2} .
$$

For a $p$-polarized source and a sensor which detects only s-polarized light

$$
Q=Q_{p s}=\left|\frac{(\varepsilon-1) \sqrt{\varepsilon-\sin ^{2} \theta_{i}} \sin \varphi_{s}}{\left(\varepsilon \cos \theta_{i}+\sqrt{\varepsilon-\sin ^{2} \theta_{i}}\right)\left(\cos \theta_{s}+\sqrt{\varepsilon-\sin ^{2} \theta_{s}}\right)}\right|^{2} .
$$

For a s-polarized source and a sensor which detects only p-polarized light 


$$
Q=Q_{s p}=\left|\frac{(\varepsilon-1) \sqrt{\varepsilon-\sin ^{2} \theta_{s}} \sin \varphi_{s}}{\left(\cos \theta_{i}+\sqrt{\varepsilon-\sin ^{2} \theta_{i}}\right)\left(\varepsilon \cos \theta_{i}+\sqrt{\varepsilon-\sin ^{2} \theta_{s}}\right)}\right|^{2} .
$$

For a $p$-polarized source and a sensor which detects only $p$-polarized light

$$
Q=Q_{p p}=\left|\frac{(\varepsilon-1)\left(\sqrt{\varepsilon-\sin ^{2} \theta_{s}} \sqrt{\varepsilon-\sin ^{2} \theta_{i}} \cos \varphi_{s}-\varepsilon \sin \theta_{i} \sin \theta_{s}\right)}{\left(\varepsilon \cos \theta_{i}+\sqrt{\varepsilon-\sin ^{2} \theta_{i}}\right)\left(\varepsilon \cos \theta_{s}+\sqrt{\varepsilon-\sin ^{2} \theta_{s}}\right)}\right|^{2}
$$

For a $s$-polarized source and a sensor without polarization discrimination

$$
Q=Q_{s s}+Q_{s p}
$$

and similarly for a $p$-polarized source and a sensor without polarization discrimination

$$
Q=Q_{p s}+Q_{p s} .
$$

For an unpolarized source and a sensor which detects only s-polarized light

$$
Q=Q_{s s}+Q_{p s}
$$

and for an unpolarized source and a sensor which detects only $p$-polarized light

$$
Q=Q_{s p}+Q_{p p}
$$

If the source is unpolarized and the sensor has no polarization discrimination

$$
Q=Q_{s s}+Q_{s p}+Q_{p s}+Q_{p p}
$$

\subsection{Beckmann-Kirchhoff Surface Scatter Theory}

The Rayleigh-Rice model is valid for optically smooth surfaces $\left(\sigma_{s} \ll \lambda\right)$. To describe scattering from rougher surfaces $\left(\sigma_{s} \approx \lambda\right)$, the Beckmann-Kirchhoff model (B-K) can be used. Unfortunately, the Beckmann-Kirchhoff model is a scalar theory which does not take polarization into account. This discussion follows Beckmann's discussion found in [3]. The geometry for the Beckmann-Kirchhoff model is the same as that for the Rayleigh-Rice model shown figure 2.6. The scattered field can be found at an arbitrary point $P$ using the Helmholtz integral 


$$
\overrightarrow{E_{2}}(P)=\frac{1}{4 \pi} \int_{S}\left(\vec{E} \frac{\partial \psi}{\partial n}-\psi \frac{\partial \vec{E}}{\partial n}\right) \mathrm{d} S
$$

where

$$
\psi=\frac{\exp \left(j k_{2} R_{2}\right)}{R_{2}} .
$$

$\vec{E}$ gives the electric field on the surface, $\overrightarrow{k_{2}}$ is the propagation vector for the scattered radiation, $R_{2}$ is the distance between the point $P$ and the surface and $\partial / \partial n$ represents the derivative along the surface normal. By assuming the radius of curvature of the surface is large compared to the wavelength; Beckmann approximates the field and its derivative by those which would be incident on a plane tangent to the surface

$$
\overrightarrow{E_{2}}=(1+R) \overrightarrow{E_{1}}
$$

and

$$
\frac{\partial \overrightarrow{E_{2}}}{\partial n}=(1-R) \overrightarrow{E_{1}}\left(\overrightarrow{k_{1}} \bullet \vec{n}\right) .
$$

In these equations, $\overrightarrow{E_{1}}$ and $\overrightarrow{k_{1}}$ give the incident fields and propagation vector respectively, and $R$ is the Fresnel surface reflectance. Beckmann then introduced a scattering coefficient given by

$$
\rho=\frac{\overrightarrow{E_{2}}}{\overrightarrow{E_{20}}},
$$

where $\overrightarrow{E_{20}}$ is the field which would be reflected in the specular direction by a planar perfect reflecting surface. If $P$ is in the Fraunhofer zone, the scattering coefficient can be written as

$$
\rho=\frac{F\left(\theta_{i}, \theta_{s}, \varphi_{s}\right)}{A} \int_{A} \int \exp (j \vec{v} \bullet \vec{r}) \mathrm{d} x \mathrm{~d} y
$$

utilizing the obliquity factor $F$ given by

$$
F\left(\theta_{i}, \theta_{s}, \varphi_{s}\right)=\frac{1+\cos \theta_{i} \cos \theta_{s}-\sin \theta_{i} \sin \theta_{s} \cos \varphi_{s}}{\cos \theta_{i}\left(\cos \theta_{i}+\cos \theta_{s}\right)} .
$$

$A$ is the area of interest on the surface, $\vec{r}$ describes the position on the surface and is given by 


$$
\vec{r}=x \vec{x}+y \vec{y}+z \vec{z}
$$

and $\vec{v}$ is the difference between $\overrightarrow{k_{1}}$ and $\overrightarrow{k_{2}}$ and is given by

$$
\vec{v}=\overrightarrow{k_{1}}-\overrightarrow{k_{2}}=\frac{2 \pi}{\lambda}\left[\left(\sin \theta_{i}-\sin \theta_{s} \cos \varphi_{s}\right) \vec{x}-\left(\sin \theta_{s} \sin \varphi_{s}\right) \vec{y}-\left(\cos \theta_{i}+\cos \theta_{s}\right) \vec{z}\right]
$$

With an introduction of the variables $v_{x}, v_{y}$ and $v_{z}, \vec{v}$ can be written as the sum of its components

$$
\vec{v}=v_{x} \vec{x}+v_{y} \vec{y}+v_{z} \vec{z}
$$

If the exact surface height function $h(x, y)$ is unknown, it can be described with a probability density $p(z)$, where $z$ is the surface height at point $(x, y)$. The joint probability density is $p\left(z_{1}, z_{2}\right)$, where $z_{1}$ and $z_{2}$ are the heights at the points $\left(x_{1}, y_{1}\right)$ and $\left(x_{2}, y_{2}\right)$. If $h(x, y)$ is stationary, it will not depend on $\left(x_{1}, y_{1}\right)$ and $\left(x_{2}, y_{2}\right)$, but only the distance between them. The characteristic function for $p(z)$ is given by [12]

$$
\chi(t)=\langle\exp (j t z)\rangle=\int_{-\infty}^{\infty} \int_{-\infty}^{\infty} p(z) \exp (j t z) \mathrm{d} z
$$

Likewise, the characteristic function for $p\left(z_{1}, z_{2}\right)$ is given by [12]

$$
\chi_{2}\left(t_{1}, t_{2}\right)=\left\langle\exp \left(j t_{1} z_{1}+j t_{2} z_{2}\right)\right\rangle=\int_{-\infty}^{\infty} \int_{-\infty}^{\infty} p\left(z_{1}, z_{2}\right) \exp \left(j t_{1} z_{1}+j t_{2} z_{2}\right) \mathrm{d} z_{1} \mathrm{~d} z_{2} .
$$

Constraining the area of interest, $A$, to $-X$ to $X$ in the $x$-direction and $-Y$ to $Y$ in the $y$ direction, the mean scattered field in any direction is given by

$$
\langle\rho\rangle=\frac{F}{A} \int_{-X}^{X} \int_{-Y}^{Y} \exp \left(j v_{x} x+j v_{y} y\right)\left\langle\exp \left(j v_{z} z\right)\right\rangle \mathrm{d} x \mathrm{~d} y
$$

which is equivalent to

$$
\langle\rho\rangle=\frac{F}{A} \chi\left(v_{z}\right) \int_{-X}^{X} \int_{-Y}^{Y} \exp \left(j v_{x}+j v_{y}\right) \mathrm{d} x \mathrm{~d} y .
$$

After integration, equation 2.41 becomes 


$$
\langle\rho\rangle=\frac{F}{A} \chi\left(v_{z}\right) \frac{\sin \left(v_{x} X\right)}{v_{x} X} \frac{\sin \left(v_{y} Y\right)}{v_{y} Y} .
$$

According to Beckmann, the mean square of the scattering coefficient is proportional to the mean scattered intensity. Finding the mean square of equation 2.42 yields

$$
\left\langle|\rho|^{2}\right\rangle=\left\langle\rho \rho^{*}\right\rangle=\left\langle\left|\frac{E_{2}}{E_{20}}\right|^{2}\right\rangle \text {. }
$$

After combining equations 2.33 and 2.39, and transforming the result to polar coordinates

$$
\left\langle\rho \rho^{*}\right\rangle=\frac{2 \pi F^{2}}{A} \int_{0}^{\infty} J_{0}\left(v_{x y} \tau\right) \chi_{2}\left(v_{z},-v_{z}\right) \tau \mathrm{d} \tau
$$

where

$$
v_{x y}=\sqrt{v_{x}^{2}+v_{y}^{2}} .
$$

The mean square from equation 2.44 can also be written in terms of $\langle\rho\rangle$ and the variance of $\rho$, which Beckmann denotes as $D\{\rho\}[3,12]$

$$
\left\langle\rho \rho^{*}\right\rangle=D\{\rho\}+\langle\rho\rangle\left\langle\rho^{*}\right\rangle \text {. }
$$

The variance of $\rho$ in equation 2.46 represents the diffuse portion of the scattered beam, while $\langle\rho\rangle\left\langle\rho^{*}\right\rangle$ represents the specular portion. After combining equations 2.42, 2.44 and 2.46 , the diffusely scattered radiation can be written as

$$
D\{\rho\}=\frac{2 \pi F^{2}}{A} \int_{0}^{\infty} J_{0}\left(v_{x y} \tau\right)\left[\chi_{2}\left(v_{z},-v_{z}\right)-\chi\left(v_{z}\right) \chi^{*}\left(v_{z}\right)\right] \tau \mathrm{d} \tau .
$$

To calculate $D\{\rho\}$, one needs the characteristic functions $\chi$ and $\chi_{2}$, which are found from the probability density function $p\left(z_{1}, z_{2}\right)$, as well as the surface ACV. The surface $\mathrm{ACV}$ provides valuable information about the density of the surface features, namely the autocovariance length, $l_{c}$.

\subsection{Microfacet Scattering Model}

The Microfacet scattering model (MF) used here[15] is essentially an extension of the model of Ashikhmin et al [16]. Whereas the previous two scattering models dealt with 


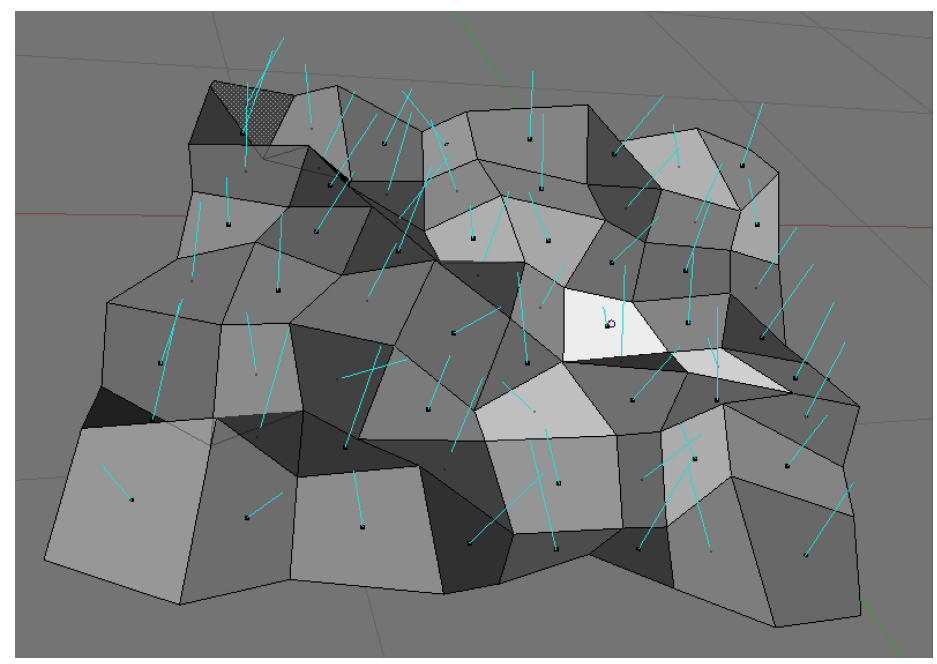

Figure 2.7: Example microfacet surface. The blue lines represent the surface normals of each microfacet.[15]

surface structures much smaller than or on the order of the the wavelength used, the microfacet model ignores these effects. The surface geometry is modeled as a ensemble of microfacets at scales much larger than the illuminating wavelength, as shown in figure 2.7.

The BRDF, defined by equation 2.15 , is given by

$$
\operatorname{BRDF}\left(\vec{k}, \overrightarrow{k^{\prime}}\right)=\frac{\mathrm{d} L^{\prime}}{\mathrm{d} E}
$$

where the primed notation indicates scattered radiation and the unprimed notation indicates incident radiation. The incident irradiance and scattered radiance can be found using their relationship to the incident and scattered flux. The flux incident on a surface area element $\mathrm{d} A$ is given by

$$
\mathrm{d} \Phi=\mathrm{d} E(\vec{n} \bullet \vec{k}) \mathrm{d} A
$$

where $\vec{n}$ is the normal of surface $\mathrm{d} A$. Solving for $\mathrm{d} E$ yields

$$
\mathrm{d} E=\frac{\mathrm{d} \Phi}{(\vec{n} \bullet \vec{k}) \mathrm{d} A}
$$

The flux scattered by surface area element $\mathrm{d} A$ is given by

$$
\mathrm{d} \Phi^{\prime}=\mathrm{d} L^{\prime} \mathrm{d} A\left(\vec{n} \bullet \vec{k}^{\prime}\right) \mathrm{d} \Omega^{\prime} .
$$

Solving for $\mathrm{d} L$ ' yields 


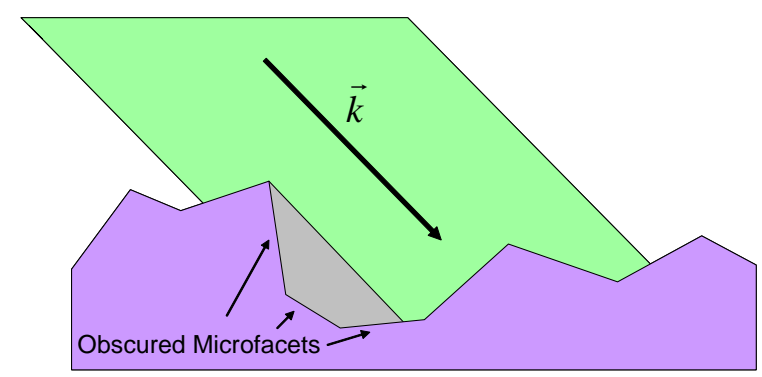

Figure 2.8: Side view of surface composed of microfacets. The green region is illuminated from direction $\vec{k}$ and the gray region is not illuminated, resulting in obscured microfacets.

$$
\mathrm{d} L^{\prime}=\frac{\mathrm{d} \Phi}{\mathrm{d} \Omega^{\prime}(\vec{n} \bullet \vec{k}) \mathrm{d} A} .
$$

When equations 2.50 and 2.52 are substituted back into equation 2.48 , the result is

$$
\operatorname{BRDF}\left(\vec{k}, \overrightarrow{k^{\prime}}\right)=\left(\frac{\mathrm{d} \Phi^{\prime}}{\mathrm{d} \Phi}\right)\left(\frac{\vec{n} \bullet \vec{k}}{\vec{n} \bullet \overrightarrow{k^{\prime}}}\right) \frac{1}{\mathrm{~d} \Omega^{\prime}} .
$$

The incident power can be written

$$
\mathrm{d} \Phi=\mathrm{d} E(\vec{n} \bullet \vec{k}) A
$$

where $A$ is the area of interest on the surface and $\vec{n}$ now refers to the bulk or average normal of the microfacets in area $A$. The scattered power can be written

$$
\mathrm{d} \Phi^{\prime}=\mathrm{d} E N_{\text {active }} A_{M F}(\vec{h} \bullet \vec{k}) F(\vec{h}, \vec{k})
$$

where $N_{\text {active }}$ is the number of microfacets oriented to allow reflection in the direction of $\vec{k}, A_{M F}$ is the area of a single microfacet and $F(\vec{h}, \vec{k})$ represents the Fresnel reflection term. The number of active microfacets is given by

$$
N_{\text {active }}=N\left[\rho(\vec{h}) \mathrm{d} \Omega_{h}\right] P\left(\vec{k} \mid \overrightarrow{k^{\prime}}\right)
$$

where $N$ is the total number of microfacets in the area $A, \rho(\vec{h})$ is the probability that a microfacet is oriented correctly, $d \Omega_{h}=d \Omega^{\prime} / 4(\vec{k} \bullet \vec{h})$ and $P\left(\vec{k} \mid \overrightarrow{k^{\prime}}\right)$ is the joint probability that a microfacet is visible from both the $\vec{k}$ and $\overrightarrow{k^{\prime}}$ direction. This probability arises from the fact that in a three dimensional surface, some microfacets may obscure others as shown in figure 2.8 . 
Substituting equations 2.54 and 2.55 into 2.53 yields the general expression for the BRDF

$$
\operatorname{BRDF}\left(\vec{k}, \overrightarrow{k^{\prime}}\right)=\frac{1}{4}\left(\frac{N A_{M F}}{A}\right)\left(\frac{\rho(\vec{h})}{\vec{n} \bullet \vec{k}}\right) F\left(\vec{k}, \overrightarrow{k^{\prime}}\right) P\left(\vec{k} \mid \overrightarrow{k^{\prime}}\right)
$$

\subsection{Modeling the Atmosphere}

Because the transmitter and receiver are both ground based, the effects of the atmosphere must be included in the system model. Recall that the model includes an upwards propagation through the atmosphere and the downwards propagation of the backscattered radiation.

The atmosphere is comprised of randomly distributed regions of air of varying temperature[7]. These regions are formed by the turbulent motion of the air, caused by the periodic heating and cooling of the Earth's surface due to the diurnal cycle. The refractive index of air is sensitive to changes in temperature. This means that the refractive index will be random throughout the atmosphere. The refractive index can be described in terms of a mean value, $n_{0}$ and a random perturbation term $n_{1}(P, t)$

$$
n(P, t)=n_{0}+n_{1}(P, t)
$$

where $P$ gives the position in space and $t$ is time. In air, the mean value of the refractive index is unity. In the regime of optical wavelengths, the dependence of the perturbation term of refractive index in air is given by [7]

$$
n_{1}=n-n_{0}=7.76 * 10^{-7} \frac{P}{T}
$$

where $\mathrm{P}$ is the air pressure in millibars and $\mathrm{T}$ is the air temperature in Kelvins.

Differentiating equation 2.59 with respect to pressure gives

$$
\frac{\mathrm{d} n_{1}}{\mathrm{~d} P}=\frac{7.76 * 10^{-7}}{T}
$$

and differentiating with respect to temperature gives

$$
\frac{\mathrm{d} n_{1}}{\mathrm{~d} T}=-7.76 * 10^{-7} \frac{P}{T^{2}} .
$$

From equations 2.60 and $2.61, n_{1}$ is clearly more susceptible to changes in temperature. Regions of air that share a uniform refractive index are called turbulent eddies. The largest of the eddies correspond to the outer scale of turbulence, $L_{o}$, which ranges between 2 and 100 meters. The smallest eddies correspond to the inner scale of turbulence, $l_{o}$, which is 
on the order of a few millimeters [14]. The spatial power spectral density of $n_{1}$ is $\Phi_{n}(\vec{\kappa})$, where $\vec{\kappa}$ is the spatial wavenumber vector and can be written as a sum of its components

$$
\vec{\kappa}=\kappa_{x} \vec{x}+\kappa_{y} \vec{y}+\kappa_{z} \vec{z} .
$$

$\Phi_{n}(\vec{\kappa})$ is a measure of the relative abundances of eddies of the sizes $l_{x}=2 \pi / \kappa_{x}$, $l_{y}=2 \pi / \kappa_{y}$ and $l_{z}=2 \pi / \kappa_{z}$. Inside of the eddies, the turbulence may be assumed to be homogeneous and isotropic and $\Phi_{n}(\vec{\kappa})$ is a function of the scalar wavenumber given by

$$
\kappa=\sqrt{\kappa_{x}^{2}+\kappa_{y}^{2}+\kappa_{z}^{2}} .
$$

For $\kappa$ in the range of $2 \pi / L_{o} \leq \kappa \leq 2 \pi / l_{o}$, Kolmogorov theory gives a PSD of the form

$$
\Phi_{n}^{K}(\vec{\kappa})=0.033 C_{n}^{2} \kappa^{-11 / 3}
$$

where $C_{n}^{2}$ is the structure constant of the reflective index perturbations and has the units of $m^{-2 / 3}$. The value of $C_{n}^{2}$ often depends on altitude. The particular atmospheric model used in this simulation uses the piecewise structure function

$$
C_{n}^{2}(h)=\left\{\begin{array}{cc}
10^{\left(-9.4010-1.5913 h-0.0606 h^{2}\right)} & 3.05 k m \leq h \leq 4.2 k m \\
10\left\{-17.1273-0.0332 h-0.0015 h^{2}+\exp \left[-\frac{1}{2}\left(\frac{15.0855-h}{5.2977}\right)^{2}\right]\right\} & 4.2 k m<h<20 k m
\end{array}\right.
$$

where $h$ is the altitude in $\mathrm{km}$ above sea level. This structure function corresponds to the night sky of Maui, Hawaii at a ground altitude of $3.08 \mathrm{~km}$. The upper extent of the atmosphere is set at an altitude of $20 \mathrm{~km}$, above which is free space.

When the telescope is looking directly upwards, $h$ is equal to $z$, the distance from the telescope. If the telescope is set at an angle $\theta_{z}$ to the vertical or zenith direction, $h$ can be found by

$$
h=z \cos \theta_{z}
$$

After finding the structure function at each layer, the atmospheric seeing or Fried parameter can be calculated. The seeing for the $i$ th layer is given by

$$
r_{0_{i}}=0.185\left[\frac{4 \pi^{2}}{k^{2} C_{n_{i}}^{2} \sec \theta_{z}}\right]^{3 / 5}
$$

With the Fried parameter known for each level, the individual phase screens are generated using the MatLab m-file phzscrn from the Adaptive Optics Toolbox developed by the Optical Sciences Company [21]. The desired output is an $n$ by $n$ array of values comprising the phase adjustment at each location, where $n$ is the side length of the sampled system 


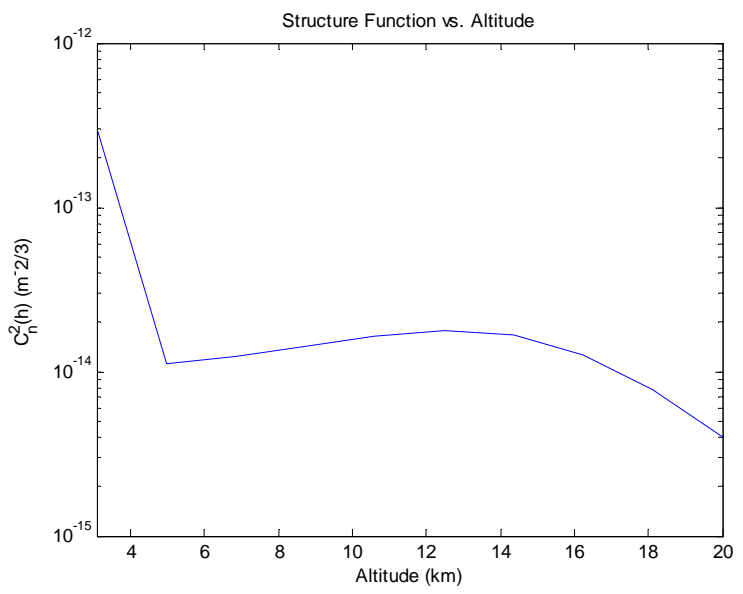

Figure 2.9: Structure function as a function of altitude for the night sky of Maui, Hawaii

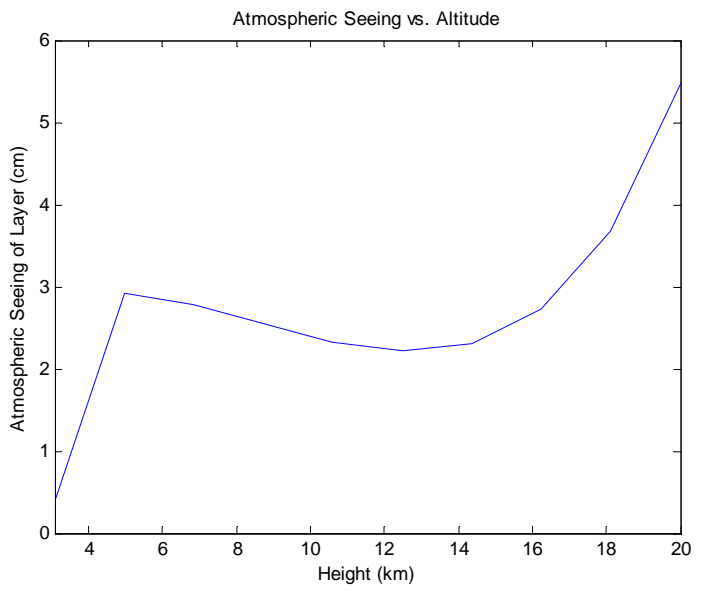

Figure 2.10: Layer atmospheric seeing as a function of altitude for the night sky of Maui, Hawaii 

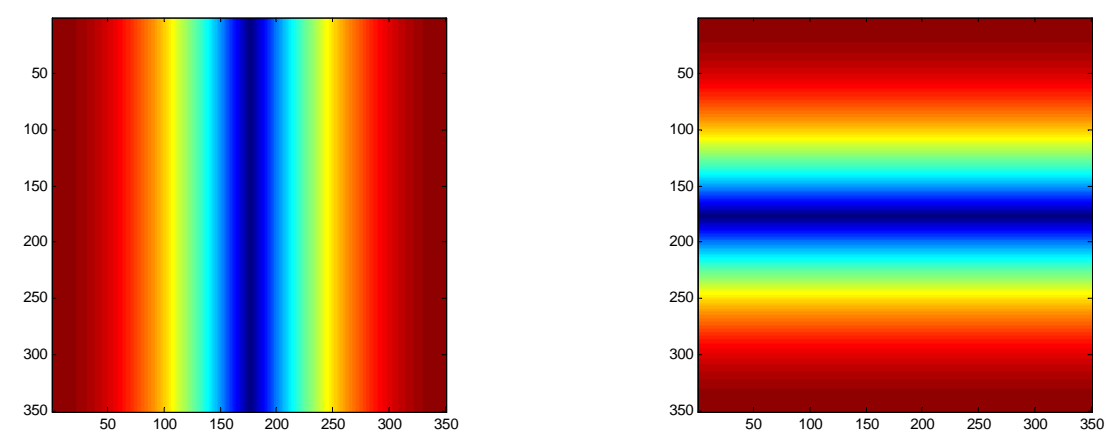

Figure 2.11: The 2-D cusp-like arrays for the $x$ and $y$ direction respectively.

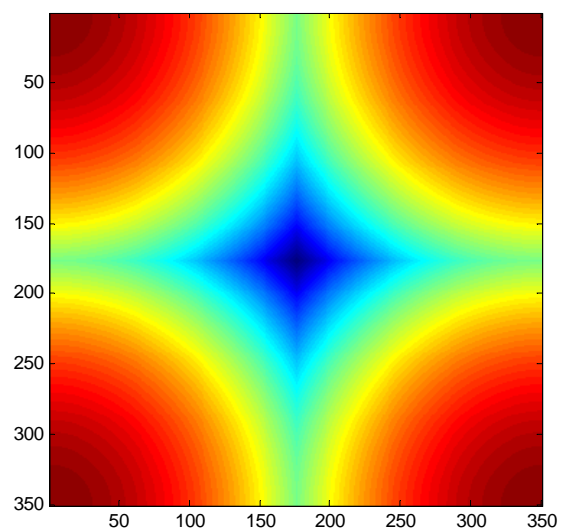

Figure 2.12: $R^{2}(x, y)$, the sum of the two cusp arrays.

array in pixels. The first step in the generation of phase screens is the creation of two 2-D cusp-like functions, as shown in figure 2.11. These functions give the square of the distance from the edges of the sample space in the $x$ direction or $y$ direction, measured in pixels.

The purpose of these cusp arrays is the creation of $R^{2}(x, y)$, shown in figure 2.12, which gives the sum of the two cusp arrays at each $(x, y)$ position. This means that the center point of $R^{2}(x, y)$ will take the value $2 n^{2}$. The base form of the phase screen can now be found with

$$
\psi=\exp \left[\log _{10}\left\{0.1517 n^{2}\left(\frac{l}{r_{0}}\right)^{5 / 6}\right\}-\frac{11}{12} \log _{10}\left\{R^{2}(x, y)\right\}\right]
$$

where $r_{0}$ is the seeing for the layer found with equation 2.67, and $n$ and $l$ are the side length 

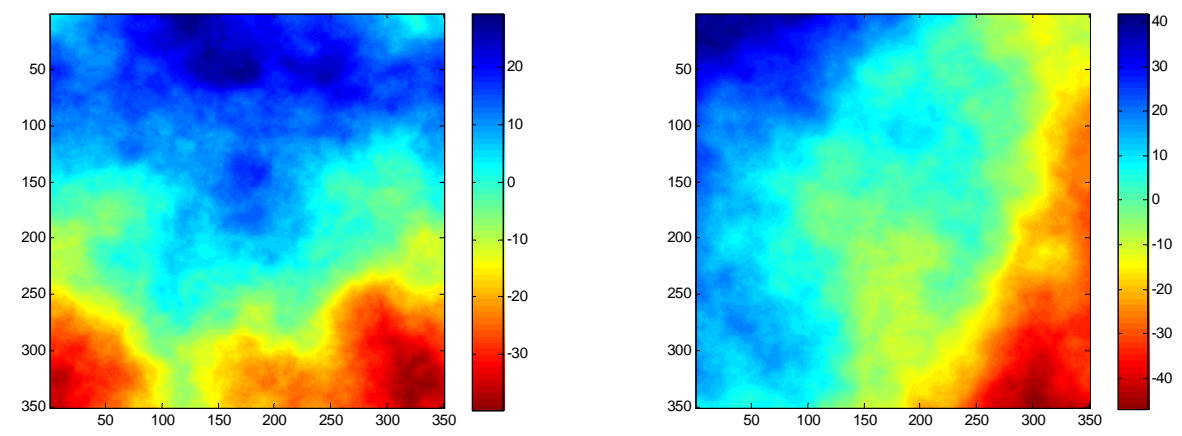

Figure 2.13: Example phase screens with $n=350, l=14 \mathrm{~m}$ and $r_{0}=.4144 \mathrm{~cm}$

of the array in pixels and meters respectively. At this point, the randomness inherent in the atmosphere is taken into account. The MatLab command randn is used to create a random number from a Gaussian distribution with a mean value of 1 and a standard deviation of 1. Each point on the base screen is multiplied by a different random complex number such that

$$
\psi_{m n}^{\prime}=\psi_{m n} *(\operatorname{rand} n+j \operatorname{rand} n) .
$$

The actual phase screen is then found by taking the Fourier transform of the adjusted base form

$$
\Psi(x, y)=\mathscr{F}\left\{\psi^{\prime}\right\} .
$$

The interaction of the optical field and the phase screen is quite simple. If the optical field immediately before the phase screen is $U(x, y)$ the field immediately after the phase screen is given by

$$
U^{\prime}(x, y)=U(x, y) * \exp [\Psi(x, y)]
$$

\subsection{Propagation Model}

The system model represents a series of laser pulses which propagate vertically between a ground-based transmitter/receiver and a scattering target in orbit at an altitude of $500 \mathrm{~km}$. The laser pulses are modeled by a 350 by 350 array with a sample spacing of $4 \mathrm{~cm}$, representing a total size of 14 by 14 meters. To properly sample across the transmitting aperture, the sample spacing is given by 


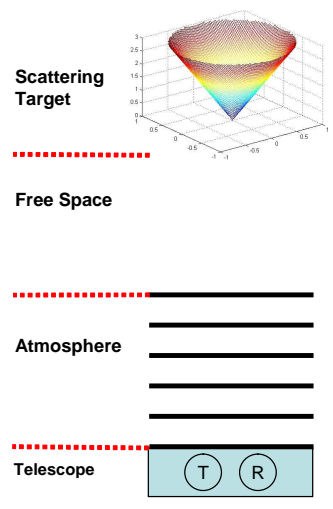

Figure 2.14: System model illustrating atmosphere and target in orbit (Not to scale)

$$
\Delta x \leq \frac{\lambda z}{2 R}
$$

where $R$ is the transmitter radius and $z$ is the propagation distance. For a propagation distance of $500 \mathrm{~km}$ and a transmitter radius of $.3 \mathrm{~m}$, a sample spacing of $4 \mathrm{~cm}$ is well under the limit presented in equation 2.72. The period of the laser pulses is $5 \mathrm{~ms}$, which is greater than the time it takes to propagate to the target and return. For this reason, the pulses are independent and do not interfere. The pulses originate from a circular aperture, with radius $r_{\text {trans }}$ and described by the pupil function $P(x, y)$, and are collected by another circular aperture upon return. The laser used is in the TEM 00 mode. The field exiting the pupil for a TEM 00 mode laser is given by

$$
U(x, y)=\exp \left(-\frac{x^{2}+y^{2}}{w^{2}}\right) P(x, y)
$$

where $w$ is the beam waist given by

$$
w=\frac{r_{\text {trans }}}{1.224} .
$$

The atmosphere is modeled as a series of ten equidistant Kolmogorov phase screen separated by $1.88 \mathrm{~km}$ of free space, for a total atmospheric thickness of $16.92 \mathrm{~km}$. A propagation distance of $1.88 \mathrm{~km}$ fulfills the Fresnel condition from equation 2.4, but fails to fulfill the more stringent Fraunhofer condition from equation 2.11. For this reason, Fourier diffraction is used to propagate between phase screens and also between the top of the atmosphere and the scattering target. To find the optical field $U(x, y ; z)$ a distance $z$ from the field $U_{0}(\xi, \eta ; z=0)$ the following is used [1] 

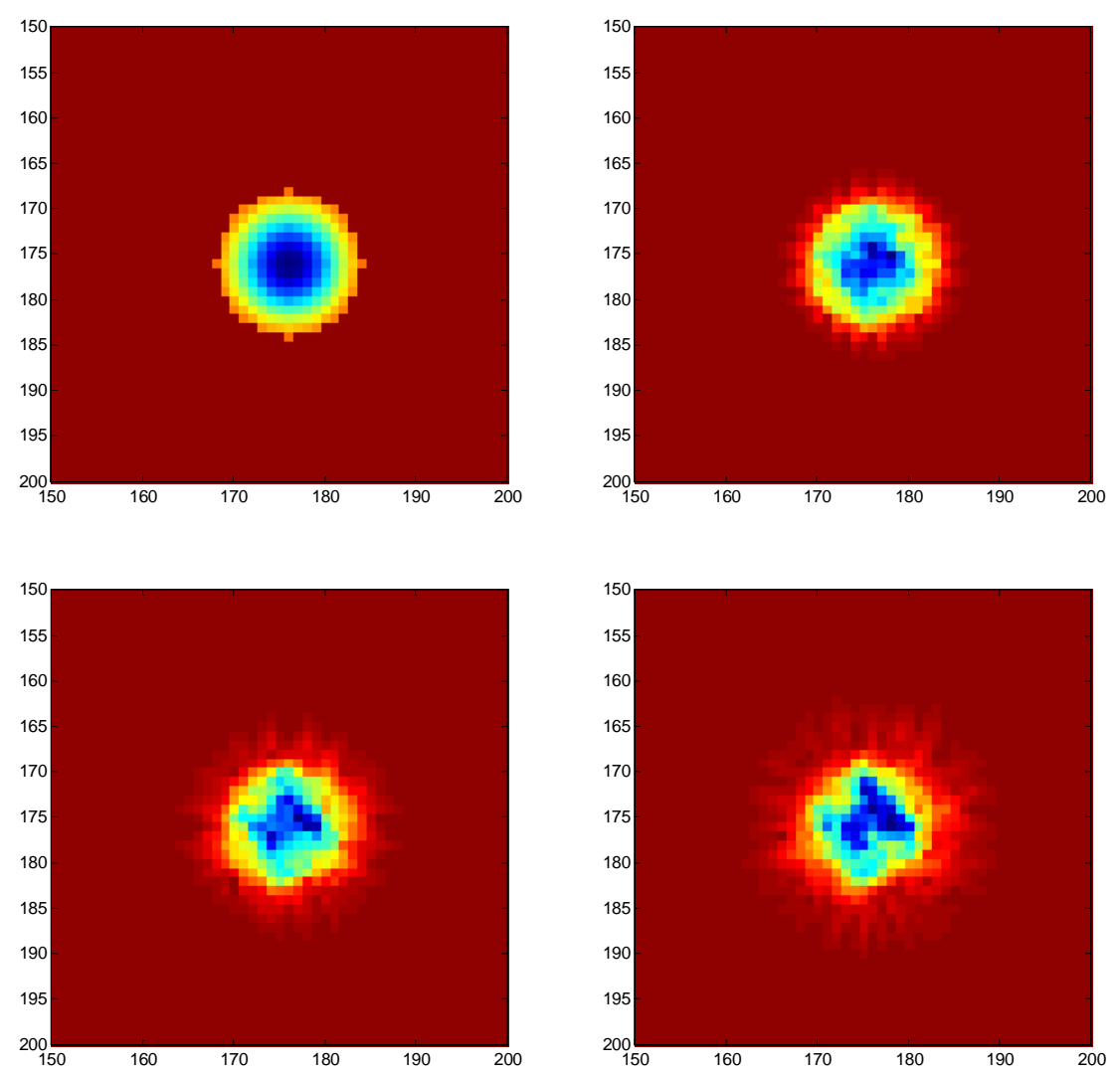

Figure 2.15: Four images of the absolute value of the optical field propagating through the 10 phase screen atmospheric model. Top Left: Field exiting transmitter. Top Right: Field after 2 phase screens. Lower Left: Field after 6 phase screens. Lower Right: Field after 10 phase screens. This is the field that will be sent to the target.

$$
U(x, y ; z)=\frac{\exp (j k z)}{j \lambda z} \exp \left[\frac{j k}{2 z}\left(x^{2}+y^{2}\right)\right] \int_{-\infty}^{\infty} \int_{-\infty}^{\infty} U_{0}(\xi, \eta) \exp \left[\frac{-2 \pi j}{h \lambda}(x \xi+y \eta)\right] \mathrm{d} \xi \mathrm{d} \eta
$$

The scattering models rely on the irradiance of the incident pulse and not the optical field. Fortunately, the irradiance of an optical field can be found using[2]

$$
E(x, y)=\varepsilon_{0} c\left\langle U(x, y)^{2}\right\rangle,
$$

where $\varepsilon_{0}$ is the permittivity of free space, and the $\langle\ldots\rangle$ operator represents a temporal average. The power incident on a surface element with area $A$ is given by [13] 


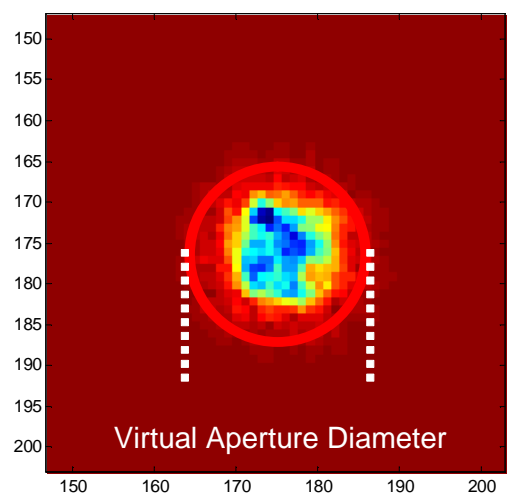

Figure 2.16: Example virtual aperture surrounding the optical field on axis. Using the statistics of the energy captured by the aperture, the SNR of the system can be found.

$$
P_{i}(x, y)=A E(x, y)
$$

\subsection{SNR of the Atmospheric Model}

One way to measure the effects of the atmosphere is the signal-to-noise ratio (SNR) of the power of the propagating beam for various path lengths. To measure the SNR, a series of virtual circular apertures, as shown in figure 2.16, are set up along the path of the beam. The apertures are placed directly after each of the phase screens.

Over many iterations, using different atmospheres, the energy that falls inside of the apertures is measured. The SNR is found by dividing the mean collected energy by the standard deviation of the collected energy

$$
\mathrm{SNR}=\frac{\mu_{E}}{\sigma_{E}}
$$

As expected, as the pulse propagates through the atmosphere, the SNR will decrease. The SNR is also larger for greater virtual aperture diameters. The SNR at the target can be found by placing another aperture in the target plane. 


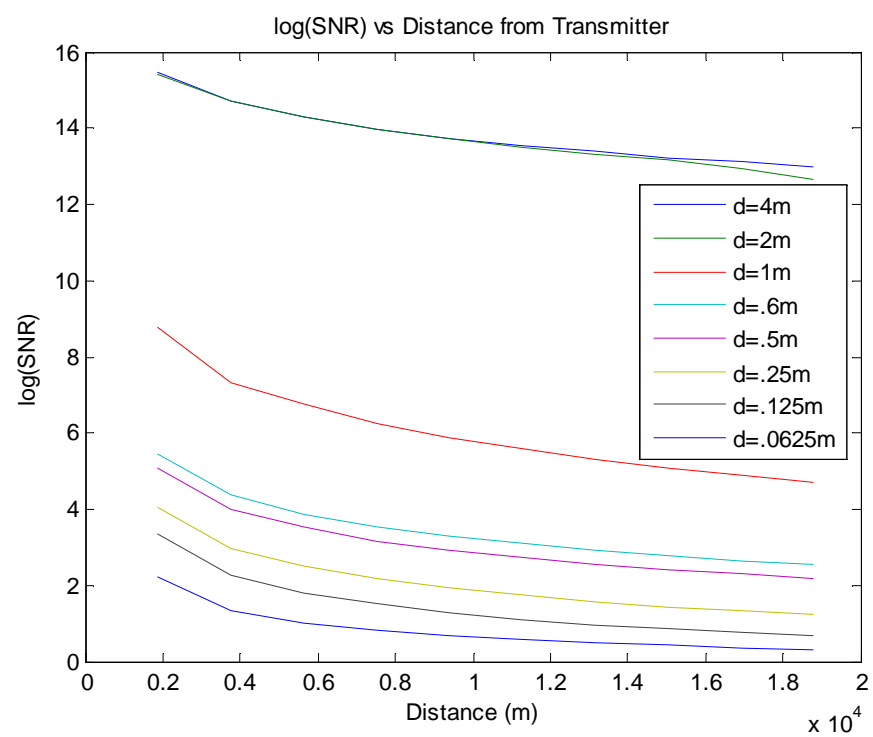

Figure 2.17: Logarithm of the SNR as a function of propagation distance for various aperture diameters. Values found from 1000 separate atmospheric iterations with a transmitter diameter of $.6 \mathrm{~m}$.

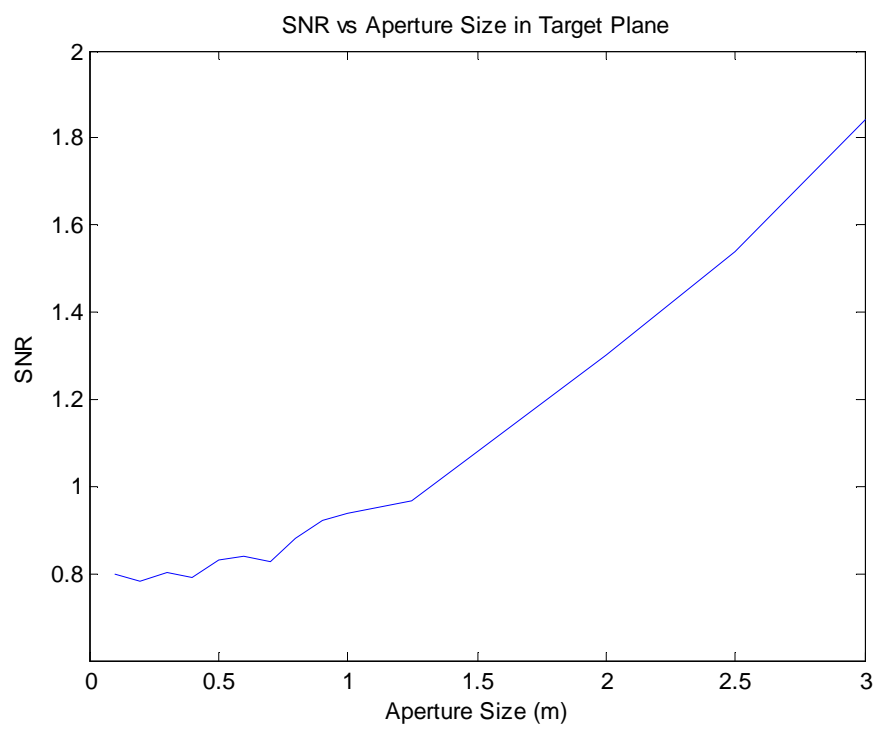

Figure 2.18: SNR as a function of aperture diameter in the target plane. Values found from 2000 separate atmospheric iterations with a transmitter diameter of .6 m. 


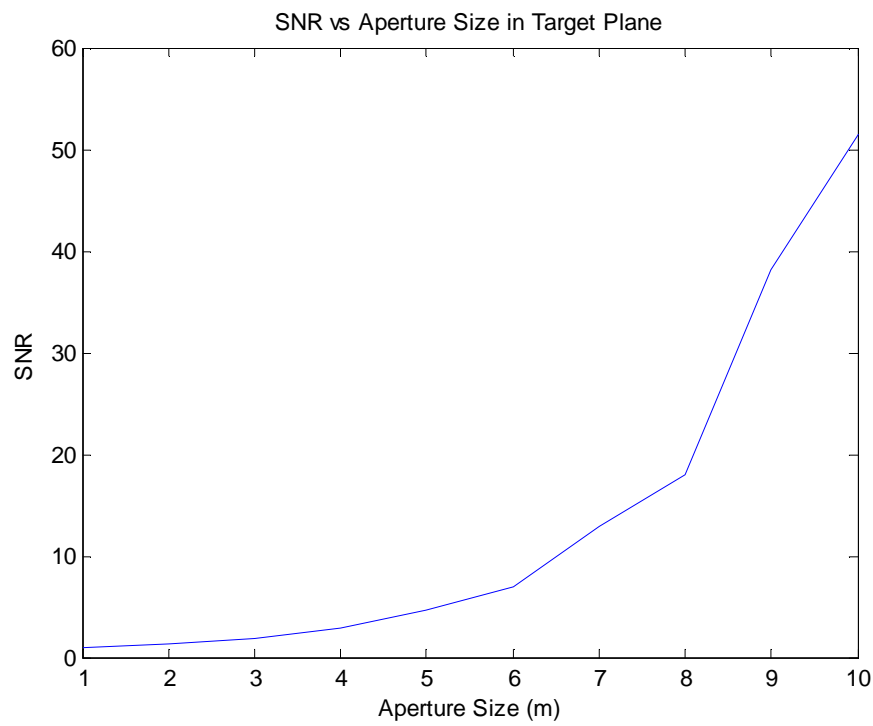

Figure 2.19: SNR as a function of aperture diameter in the target plane. Values found from 1000 separate atmospheric iterations with transmitter diameter of $.6 \mathrm{~m}$. 


\section{Chapter 3}

\section{Construction of System Model}

\subsection{Target Creation}

Both the Rayleigh-Rice and Beckmann-Kirchhoff scattering models described in Chapter 2 are continuous functions. To model scattering in a computer environment, they must be made discrete. The crucial values for creating scattering distributions are the incident and scattering angles, as well as the RMS surface roughness and autocovariance length. All of the targets used in this thesis are assumed to be isotropic, such that the RMS surface roughness and autocovariance length are constant for the entire surface.

To find the necessary incident and scattering angles, a depth map version of the target is used. The depth map is a discrete two-dimensional function of $x$ and $y$ which gives the $z$ coordinate of the visible surface boundary.

Using the depth map of the target, the important scattering angles can be found. The normal vector at any point on the target surface can be calculated by finding the cross product of any two vectors in the tangent plane. This normal vector is calculated for every point on the surface. The angle between the normal and $z$-axis is simply $\theta_{i}$. Another important angle is $\gamma$, which is measured between the normal vector and the $y$-axis as shown in figure 3.3.

The angle $\gamma$ is crucial for translating from the traditional $x-y$ polarization coordinates of the transmitter to the $s$ - $p$ coordinates defined on the target surface for the RayleighRice model. For an incoming pulse with polarization components $U_{X}$ and $U_{Y}$, the surface components $U_{S}$ and $U_{P}$ can be found using the following cosine transformations

$$
\begin{gathered}
U_{S}=U_{X} \cos \gamma+U_{Y} \cos \gamma \\
U_{P}=-U_{X} \sin \gamma+U_{Y} \cos \gamma .
\end{gathered}
$$

And conversely, after the reflection $U_{X}$ and $U_{Y}$ can be found again using the transformations 


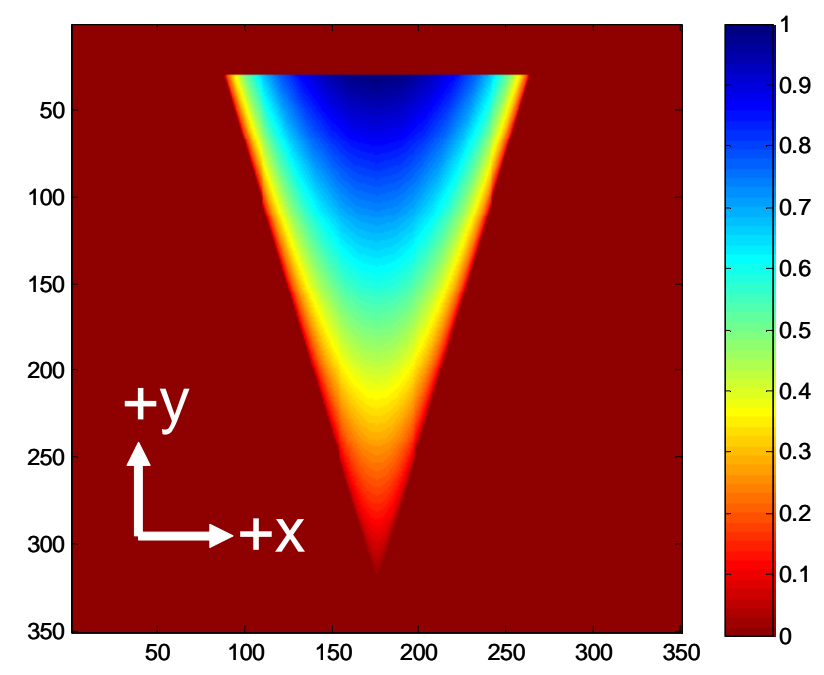

Figure 3.1: Example depth map for a conical target with coordinate description. $Z$-axis is positive out of the page. Transmitter/receiver setup is along the $z$-axis.
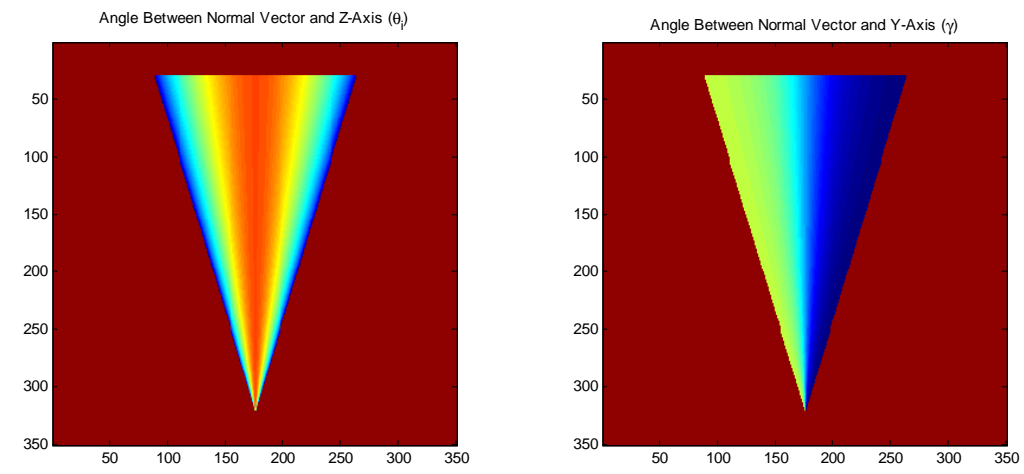

Figure 3.2: Angle found from depth map in figure 3.1. Left: Angle between normal vector and z-axis $\left(\theta_{i}\right)$ in radians. Right: Angle between normal vector and y-axis $(\gamma)$ in radians. 


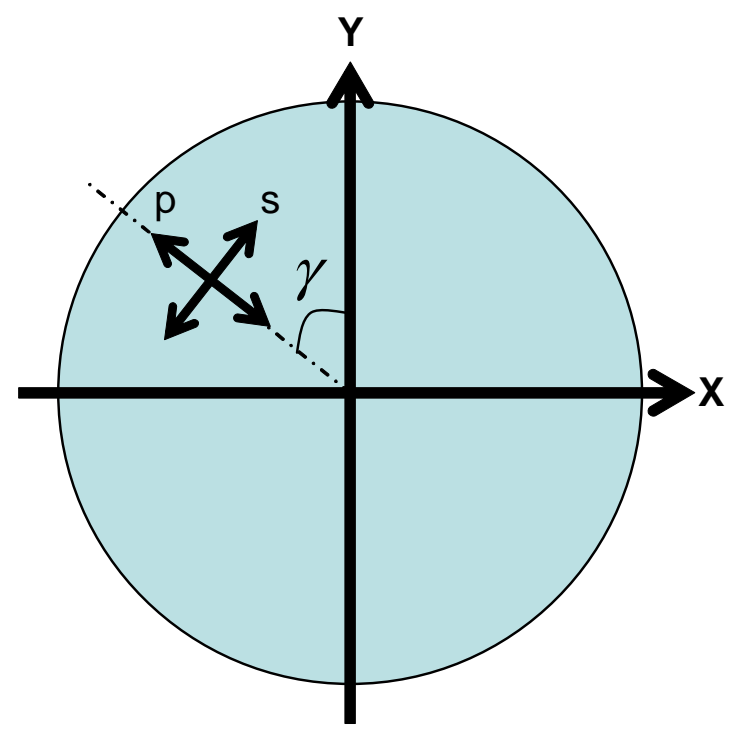

Figure 3.3: Geometry for conversion between $x-y$ polarization and $s-p$ polarization coordinates on conical target pointing along positive $z$-axis.

$$
\begin{aligned}
& U_{X}=U_{S} \cos \gamma-U_{P} \sin \gamma \\
& U_{Y}=U_{S} \sin \gamma+U_{P} \cos \gamma .
\end{aligned}
$$

Using the unique geometry of the system, a few simplifications can be made. Because the transmitter and receiver are earth-based and in close proximity, the only scattering direction of interest is back towards the direction of arrival. Using the notation introduced in figure 2.6, $\theta_{s}$ will always be equal to $\theta_{i}$ and $\varphi_{s}$ will always equal $\pi$ radians. This is shown clearly in figure 3.4 .

Because a time history of return pulses is desired, the system's time resolution is especially important at the target. For this purpose the target is divided into equal thickness slices along the optical axis. As the incoming pulse arrives at the target, it encounters each slice in succession. For each any incoming pulse, multiple pulses are scattered back towards the receiver. Originally the slice forming algorithm was very simple; all values used were either zero or one, as shown in figure 3.5. If the target boundary existed in a certain location, the slice value became unity. If the boundary did not exist in that location, the slice value became zero. Whatever scattering method used was calculated and multiplied by the slice values to give the radiation scattered from each slice.

Since the Fourier transform is used in the propagation of the pulses, the sharp contrast between zero and one led to non-physical ringing artifacts in the return pulses, see figures 


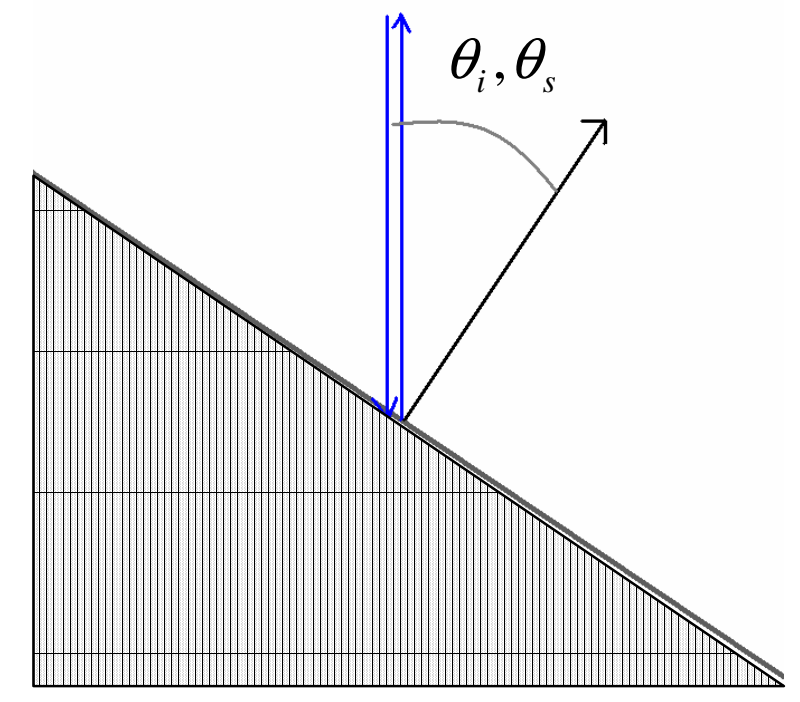

Figure 3.4: Cross-section of target. The red arrows indicate incoming and scattered radiation of interest. The black arrow shows the surface normal, from which both $\theta_{i}$ and $\theta_{s}$ are measured.
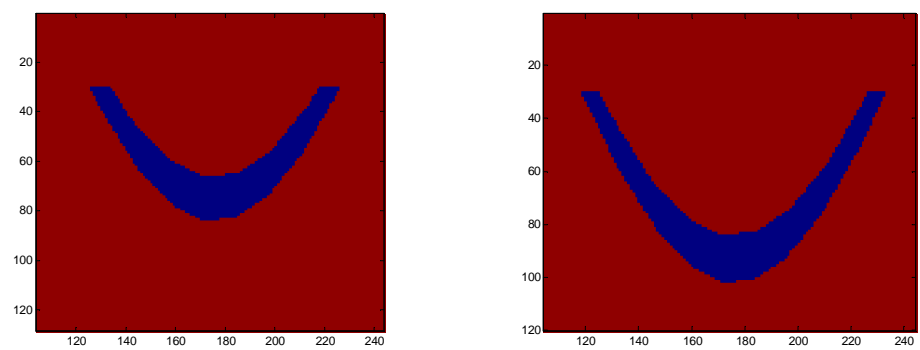

Figure 3.5: Consecutive slices of a conical target oriented pointing downward. Red values are equal to one, while blue values are equal to zero. 

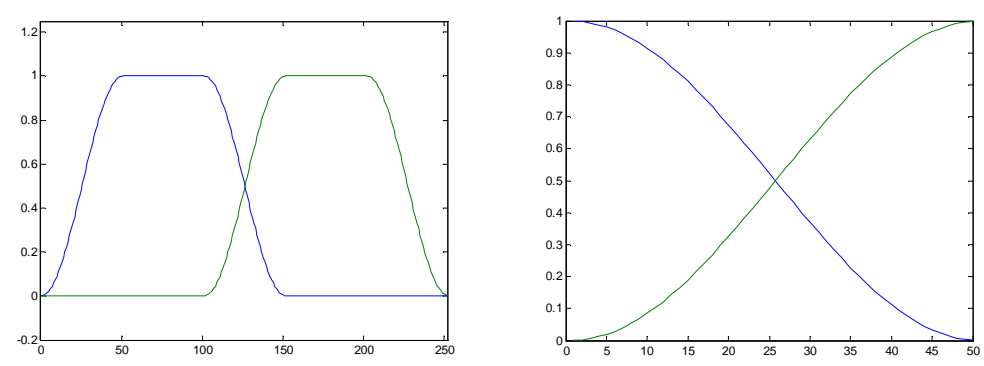

Figure 3.6: Left: Two entire Hanning slices. Right: Close up of overlapping region. At every horizontal location the sum of the two edges is equal to unity.
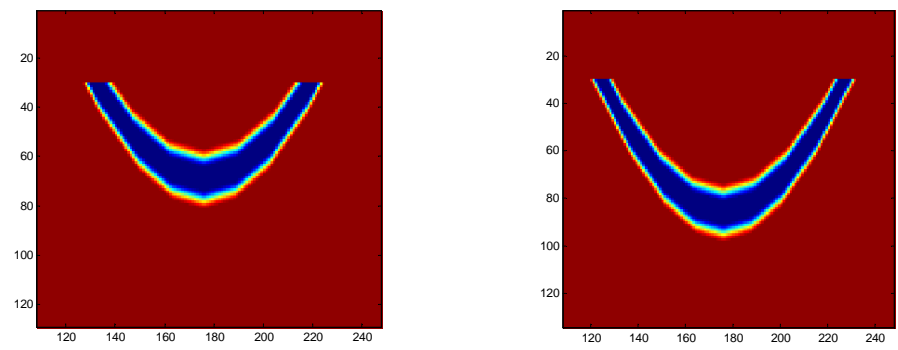

Figure 3.7: Consecutive slices of a conical target oriented pointing downward utilizing Hanning edges.

3.8 and 3.9. The solution to this problem was to remove the sharp contrast in the transition between slices. This was accomplished by adding Hanning edges to the slices[9]. The Hanning function takes the form

$$
A(x)=\frac{1}{2}\left[1+\cos \left(\frac{\pi x}{a}\right)\right],
$$

where $a$ is the full width at half maximum (FWHM). Each Hanning slice has three components, divided by depth. The region between the leading edge of the slice and one fourth of the total thickness of the slice is a Hanning function rising from 0 to 1 . Conversely, the region between three fourth of the total thickness and the trailing edge of the slice is another Hanning function falling from 1 to 0 . The region in between, which makes up half of the overall thickness of the slice, is unity. Examples of the Hanning slices are shown in figure 3.7.The unique part about these Hanning slices is the fact that when two slices overlap, their sum is equal to one. 

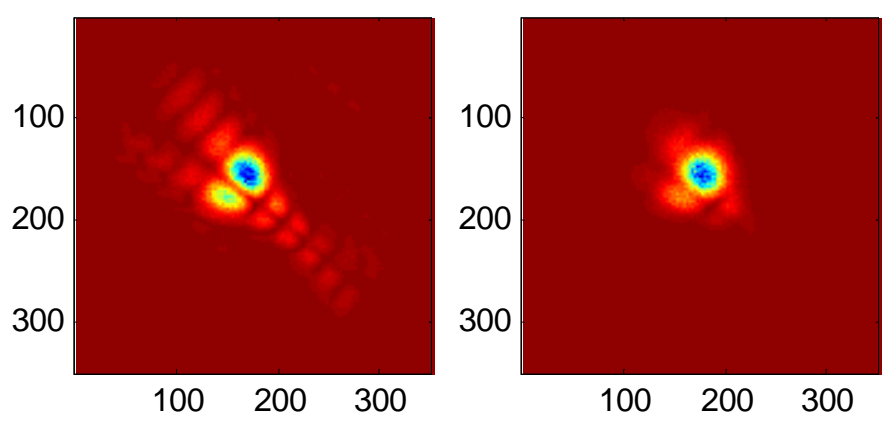

Figure 3.8: Radiation scattered back to the aperture plane from identical slices of a conical target pointing towards the top right corner of the array. The left image was constructed using the original slice forming algorithm and shows evidence of ringing. The right image was formed using slices with Hanning edges.
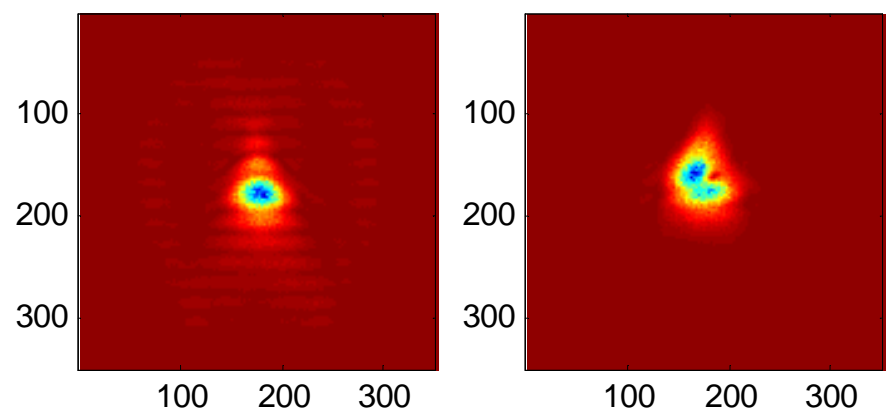

Figure 3.9: Radiation scattered back to the aperture plane from identical slices of a conical target pointing upward. The left image was constructed using the original slice forming algorithm and shows evidence of ringing. The right image was formed using slices with Hanning edges. 


\subsection{Implementation of the Rayleigh-Rice Model}

Critical to the Rayleigh-Rice model is the PSD of the target surface. Here the assumption is made that both the surface height distribution and surface ACV functions obey Gaussian distributions. In this case the standard deviations of the surface height distribution and ACV functions are the RMS surface roughness and autocovariance length respectively. Also, the assumption is made that the RMS surface roughness is isotropic. The surface ACV function is circularly symmetric and given by

$$
C(r)=\sigma_{s}^{2} \exp \left[-\left(r / l_{c}\right)^{2}\right]
$$

where $r=\sqrt{x^{2}+y^{2}}$. Since the surface PSD and the ACV function obey a Fourier transform relationship, the PSD can be found to be

$$
\operatorname{PSD}\left(f_{x}, f_{y}\right)=\pi \sigma_{s}^{2} l_{c}^{2} \exp \left[-\pi^{2} l_{c}^{2}\left(f_{x}^{2}+f_{y}^{2}\right)\right]
$$

evaluated at

$$
f_{x}=\frac{\sin \theta_{s} \cos \varphi_{s}-\sin \theta_{i}}{\lambda}
$$

and

$$
f_{y}=\frac{\sin \theta_{s} \sin \varphi_{s}}{\lambda}
$$

Using the PSD given in equation 3.7, equation 2.16 becomes

$$
I\left(\theta_{s}, \varphi_{s}\right)=P_{i}\left(\frac{16 \pi^{2} \sigma_{s}^{2} l_{c}^{2}}{\lambda}\right) \cos \theta_{i} \cos ^{2} \theta_{s} Q \exp \left[-\pi l_{c}^{2}\left(f_{x}^{2}+f_{y}^{2}\right)\right] .
$$

Using equation 3.10, the intensity as a function of scattering angle can be calculated for various values of $\sigma_{s}$ and $l_{c}$. Knowing the values of $\theta_{i}, \theta_{s}, \varphi_{s}$ and $\gamma$, the backscattered intensity can be found for every point on the target surface. All of the following distributions use $\varepsilon=.84+1.84 j$, which is the dielectric constant of gold. Scattering distributions for the Rayleigh-Rice model for various values of incident angle, surface roughness and autocovariance length are show in figures 3.10, 3.11, 3.12, and 3.13.

\subsection{Implementation of Beckmann-Kirchhoff Model}

To implement the Beckmann-Kirchhoff scattering model on a surface, the surface height distribution must be known. As with the Rayleigh-Rice model, the assumption is made that both the surface height distribution and the ACV follow Gaussian distributions. The 

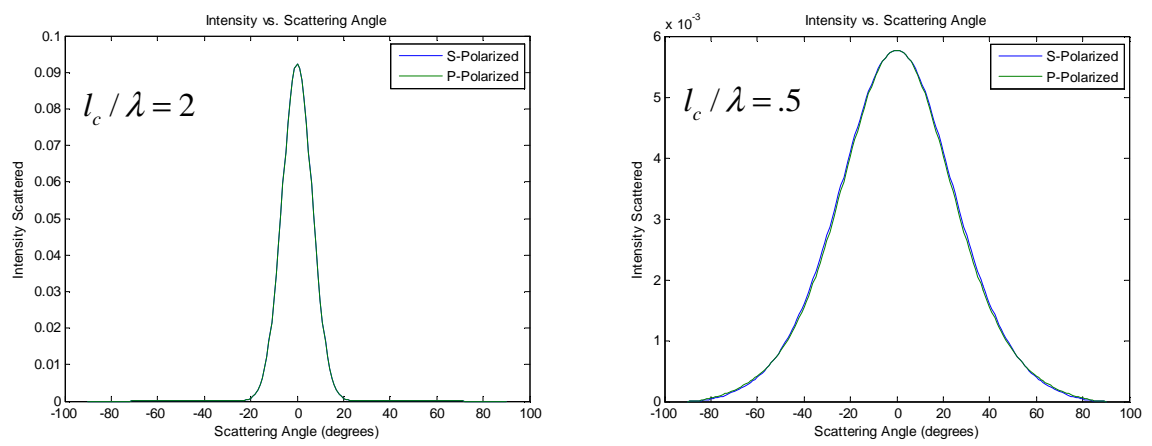

Figure 3.10: Scattering distributions using R-R model with $\sigma_{s} / \lambda=.02$ and an incident angle of $0^{\circ}$.
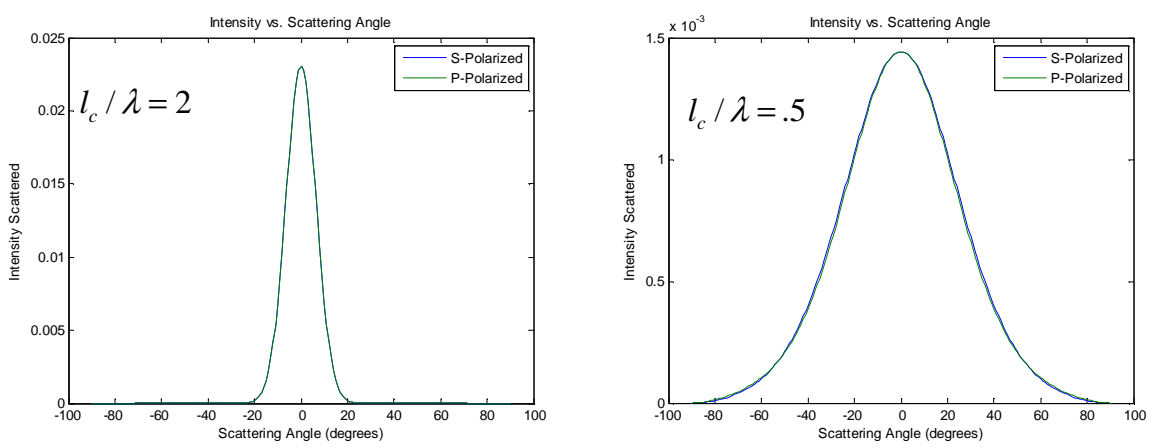

Figure 3.11: Scattering distributions using R-R model with $\sigma_{s} / \lambda=.01$ and an incident angle of $0^{\circ}$.
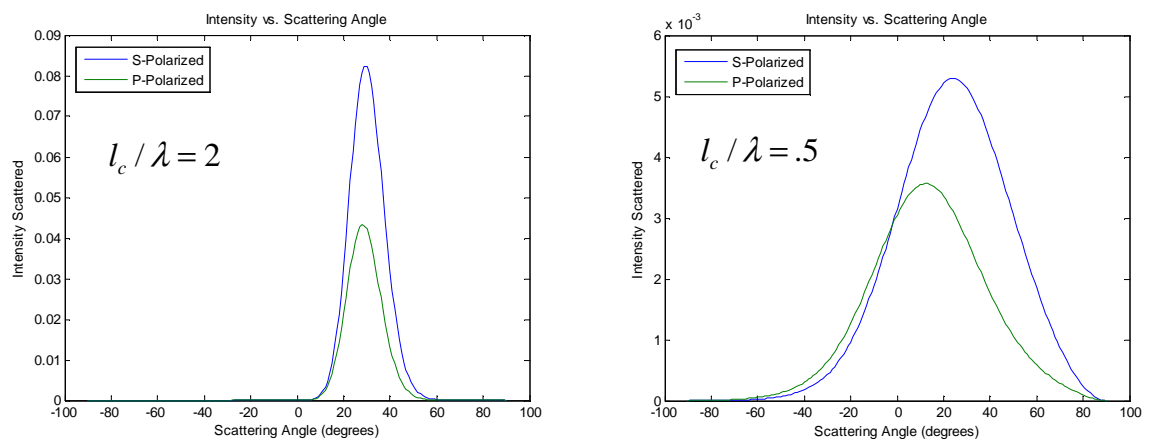

Figure 3.12: Scattering distributions using R-R model with $\sigma_{s} / \lambda=.02$ and an incident angle of $-30^{\circ}$. 

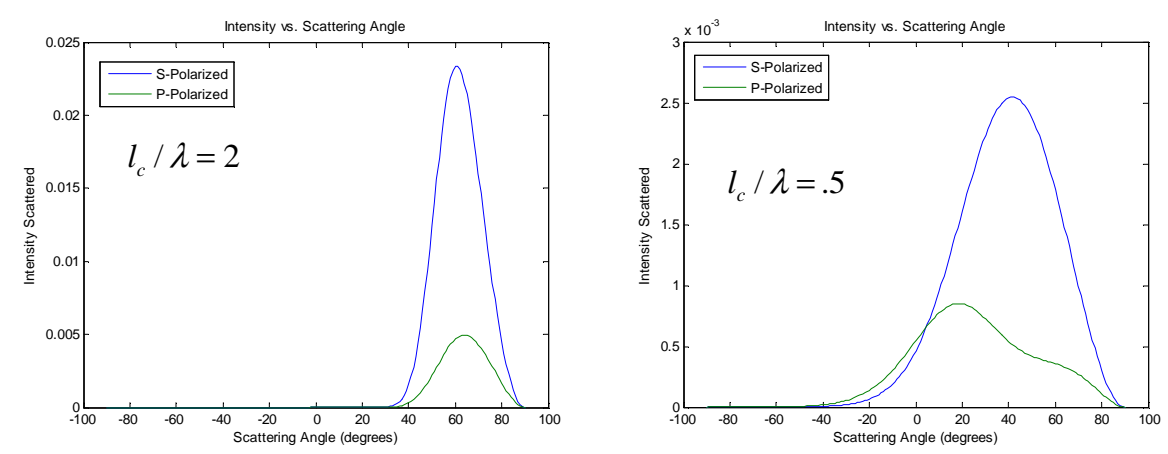

Figure 3.13: Scattering distributions using R-R model with $\sigma_{s} / \lambda=.01$ and an incident angle of $-30^{\circ}$.

joint probability density for two surface heights $h_{1}$ and $h_{2}$ which are zero mean is given by $[3,12]$

$$
p\left(z_{1}, z_{2}\right)=\frac{1}{2 \pi \sigma_{s}^{2} \sqrt{1-C^{2}}} \exp \left(-\frac{z_{1}^{2}-2 C z_{1} z_{2}+z_{2}^{2}}{2 \sigma_{s}^{2}\left(1-C^{2}\right)}\right)
$$

where $C$ is the autocovariance function

$$
C(\tau)=\exp \left(-\frac{\tau^{2}}{l_{c}^{2}}\right)
$$

In one dimension, the characteristic function associated with the Gaussian distribution is given by

$$
\chi(v)=\exp \left(-\frac{1}{2} \sigma_{s}^{2} v^{2}\right)
$$

Using the joint distribution from equation 3.11 in equation 2.39 gives the characteristic function for the surface,

$$
\chi_{2}\left(v_{z},-v_{z}\right)=\exp \left[-\sigma_{s}^{2} v_{z}^{2}(1-C)\right] .
$$

When the ACV from equation 3.12 is used in equation 3.14, and the result is expanded into a MacLaurin series, the characteristic function is given as

$$
\chi_{2}\left(v_{z},-v_{z}\right)=\exp \left(-v_{z}^{2} \sigma_{s}^{2}\right) \sum_{m=0}^{\infty} \frac{v_{z}^{2 m} \sigma_{s}^{2 m}}{m !} \exp \left(-\frac{m \tau^{2}}{l_{c}^{2}}\right) .
$$

Substituting the characteristic functions from by equations 3.13 and 3.15 into equation 2.47 gives 


$$
D\{\rho\}=\frac{2 \pi F^{2} \exp (-g)}{A} \sum_{m=1}^{\infty} \frac{g^{m}}{m !} \int_{0}^{\infty} J_{0}\left(v_{x y} \tau\right) \exp \left(-\frac{m \tau^{2}}{l_{c}^{2}}\right) \tau \mathrm{d} \tau
$$

where

$$
g=v_{z}^{2} \sigma_{s}^{2}=\left(\frac{2 \pi \sigma}{\lambda}\right)^{2}\left(\cos \theta_{i}+\cos \theta_{s}\right)^{2}
$$

and

$$
v_{x y}=\sqrt{v_{x}^{2}+v_{y}^{2}}=\left(\frac{2 \pi}{\lambda}\right) \sqrt{\sin ^{2} \theta_{i}-2 \sin \theta_{i} \sin \theta_{s} \cos \varphi_{s}+\sin ^{2} \theta_{s}} .
$$

After evaluating the integral

$$
\int_{0}^{\infty} J_{0}\left(v_{x y} \tau\right) \exp \left(-\frac{m \tau^{2}}{l_{c}^{2}}\right) \tau \mathrm{d} \tau=\frac{1}{2 m} \exp \left(-\frac{v_{x y}^{2} l_{c}^{2}}{4 m}\right)
$$

equation 3.16 becomes

$$
D\{\rho\}=\frac{\pi l_{c}^{2} F^{2} \exp (-g)}{A} \sum_{m=1}^{\infty} \frac{g^{m}}{m ! m} \exp \left(-\frac{v_{x y}^{2} l_{c}^{2}}{4 m}\right)
$$

which is the general expression for the radiance of diffusely scattered light from a surface with a Gaussian surface height distribution and a Gaussian ACV. To reduce computation time, the maximum value of $m$ in the sum in equation 3.20 is restricted to 50 , instead of infinity. Figure 3.14 shows how this sum converges, for different values of $\theta_{i}$. Using equation 3.20, the radiance as a function of scattering angle can be calculated for various values of $\sigma_{s}$ and $l_{c}$. Knowing the values of $\theta_{i}, \theta_{s}$ and $\varphi_{s}$, the backscattered radiance can be found for every point on the target surface. All of the following distributions use $\varepsilon=.84+$ $1.84 j$, which is the dielectric constant of gold. Scattering distributions for the BeckmannKirchhoff model for various values of incident angle, surface roughness and autocovariance length are show in figures 3.15, 3.16, 3.17, and 3.18.

\subsection{Implementation of Microfacet Scattering Model}

Stryjewski et al used the Blinn-Phong normal distribution to describe the microfacet distribution [15]

$$
\rho(\vec{h})=\left(\frac{m+1}{2 \pi}\right) \cos (\vec{h} \bullet \vec{n})^{m}
$$



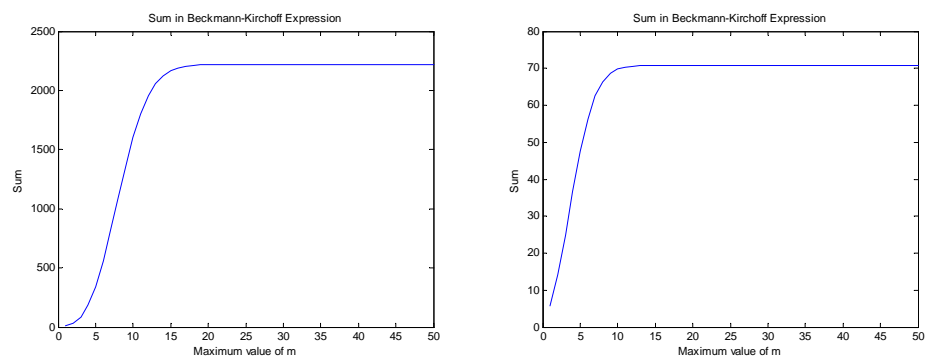

Figure 3.14: Sum in equation 3.20 as a function of maximum limit of $m$ showing convergence. In the left figure, $l_{c} / \lambda=2$ and $\theta_{i}=\theta_{s}=0^{\circ}$. In the right figure, $l_{c} / \lambda=2$ and $\theta_{i}=\theta_{s}=40^{\circ}$.
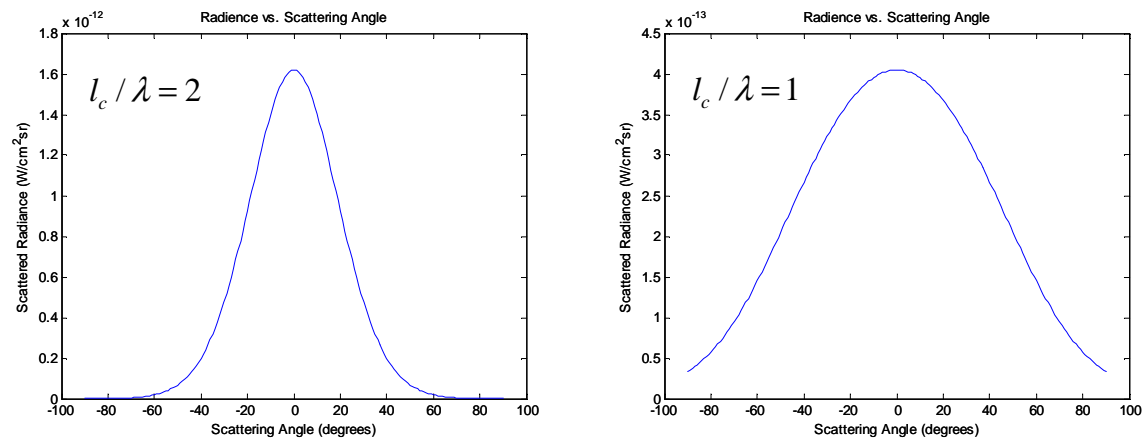

Figure 3.15: Scattering distributions using B-K model with $\sigma_{s} / \lambda=.25$ and an incident angle of $0^{\circ}$.
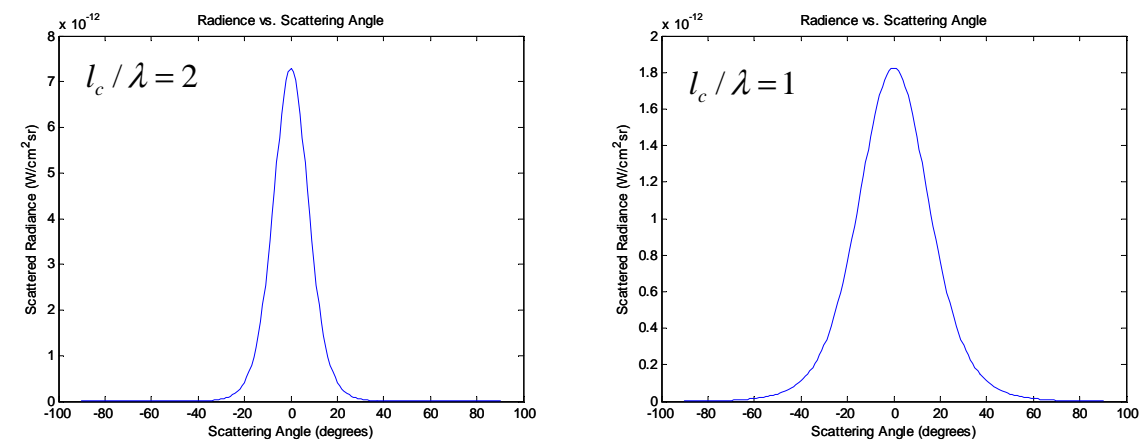

Figure 3.16: Scattering distributions using B-K model with $\sigma_{s} / \lambda=.1$ and an incident angle of $0^{\circ}$. 

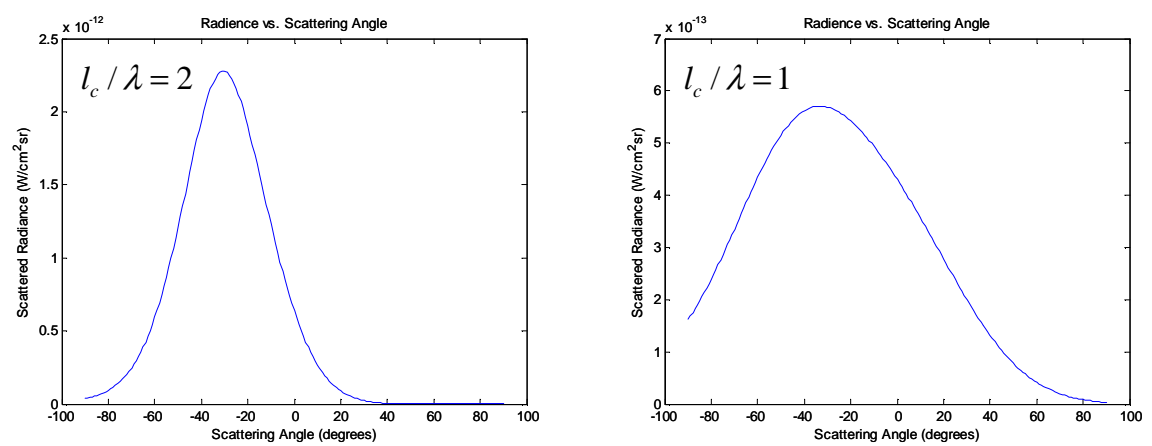

Figure 3.17: Scattering distributions using B-K model with $\sigma_{s} / \lambda=.25$ and an incident angle of $30^{\circ}$.
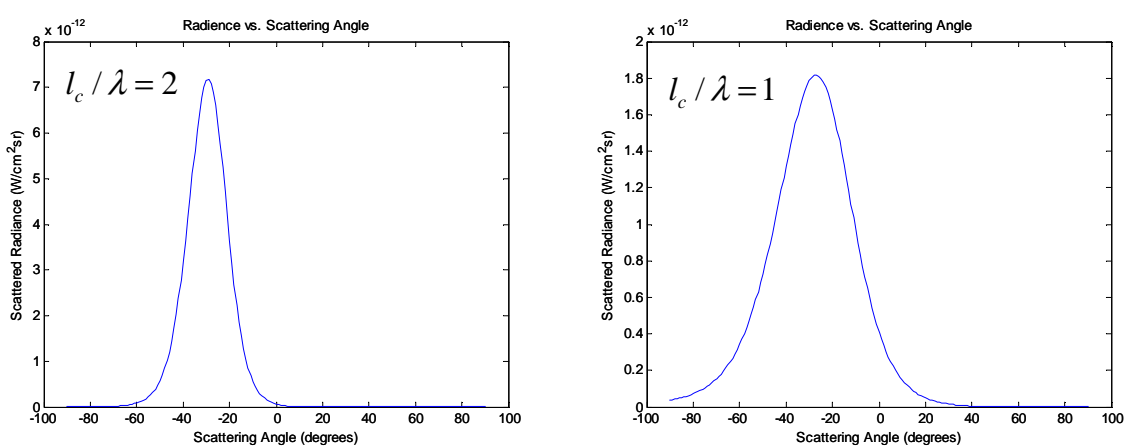

Figure 3.18: Scattering distributions using B-K model with $\sigma_{s} / \lambda=.1$ and an incident angle of $30^{\circ}$. 
where $\vec{h}$ is the local microfacet normal, $\vec{n}$ is the bulk surface normal and $m$ is a surface roughness parameter which is inversely proportional to $l_{c}$. For a Lamberian surface, which scatters equally in all directions, $m=0$. For a mirrored surface, which scatters only in the specular direction, $m=\infty$. Ashikhmin et al [16] showed that the obscuration term is given by

$$
P\left(\vec{k} \mid \overrightarrow{k^{\prime}}\right)=[1-f(\phi)] P(\vec{k}) P\left(\overrightarrow{k^{\prime}}\right)+f(\phi) \min \left\{P(\vec{k}), P\left(\overrightarrow{k^{\prime}}\right)\right\}
$$

where $f(\phi)$ is a function of $\phi$, the angle formed between $\vec{k}$ and $\vec{k}^{\prime}$ when projected on the plane normal to $\vec{n}$. Stryjewski et al [15] chose $f(\phi)=\cos ^{2}\left(\frac{\phi}{2}\right)$, which is the simplest form that remains consistent with equation 3.22. $P(\vec{k})$ is the probability that a certain microfacet will be visible from the direction $\vec{k}$ and is given by

$$
P(\vec{k})=\frac{\langle\vec{n} \bullet \vec{h}\rangle(\vec{n} \bullet \vec{k})}{\langle\vec{h} \bullet \vec{k}\rangle_{+}}
$$

where $\langle\vec{n} \bullet \vec{h}\rangle$ is the ensemble average over $\rho(\vec{h})$ and $\langle\vec{h} \bullet \vec{k}\rangle_{+}$is the ensemble average over all non-obscured microfacets, given by

$$
\langle\vec{h} \bullet \vec{k}\rangle_{+}=\frac{m+1}{\pi} \int_{(\vec{h} \bullet \vec{k})>0}(\vec{h} \bullet \vec{k}) \sin (\theta)^{m+1} \cos ^{m} \varphi \mathrm{d} \theta \mathrm{d} \varphi .
$$

This integral was evaluated by Stryjewski et al in [15]

$$
\langle\vec{h} \cdot \vec{k}\rangle_{+}=\frac{m+1}{\pi} \cos \alpha\left[2 A(m+1)-A(m+1) \cos (\alpha)^{m+2}+\tan \alpha \frac{\sin (\alpha)^{m+1}}{m+1}\right]
$$

where $\alpha$ is the angle between $\vec{k}$ and $\vec{n}$ and

$$
A(m)=\frac{\pi}{2} \frac{m !}{2^{m}\left(\frac{m}{2} !\right)^{2}} \approx \frac{\pi}{2} \frac{\sqrt{2 \pi m+1}}{m \pi+1} .
$$

The actual calculations are made using an nVIDIA GeForce 8800 GT graphics card using a shader based rendering approach in OpenGL and CUDA. The scattering is computed using the Python code LSig.py, Version 2.0.0 written by John Stryjewski, and last updated 6/24/2008. 


\section{Chapter 4}

\section{Results}

I have chosen to show scattering from two conical targets, one aligned vertically and one set at an angle to the vertical, and a rectangular prism target. The conical targets were chosen, because they display a wide array of incident angles. The rectangular prism was chosen, because it displays just two incident angles. The normal of the main face of the rectangular prism is set at an angle of $20^{\circ}$ to the optical axis. Depending on the time resolution of the system, a number of return pulses are formed from a single incident pulse. The default time resolution of the system is $d t=8 * 10^{-10} \mathrm{sec}$, which creates 15 slices, and therefore 15 return pulses, for a target with a depth of 3.5 meters. These pulses propagate back to the collecting aperture, encountering the same 10 phase screen as on the initial propagation trip. The figures in the next section are organized in groupings of eight or sixteen images. The first image is the depth map of the target as described in section 3.1. In these depth map images, red values represent surface elements that are closest to the observation point. The following images are the series of return pulses at the receiver plane.

\subsection{Backscattering from Rayleigh-Rice Surface}

With the knowledge of the incident power distribution from equation 2.77 , the scattered intensity for each slice of a Rayleigh-Rice surface can be found with equation 3.10. As shown in figure $3.4 \varphi_{s}=\pi$ and $\theta_{s}=\theta_{i}$. $\theta_{i}$ can be found from the target depth map as shown in figure 3.2. The scattered intensity is converted to scattered irradiance using [13]

$$
E_{s}(x, y)=\frac{I_{s}(x, y) \Omega}{A_{x y}}
$$

where $A_{x y}$ is the projected area of the surface element centered on $(x, y) . \Omega$ is the solid angle subtended by the detector which is given by 


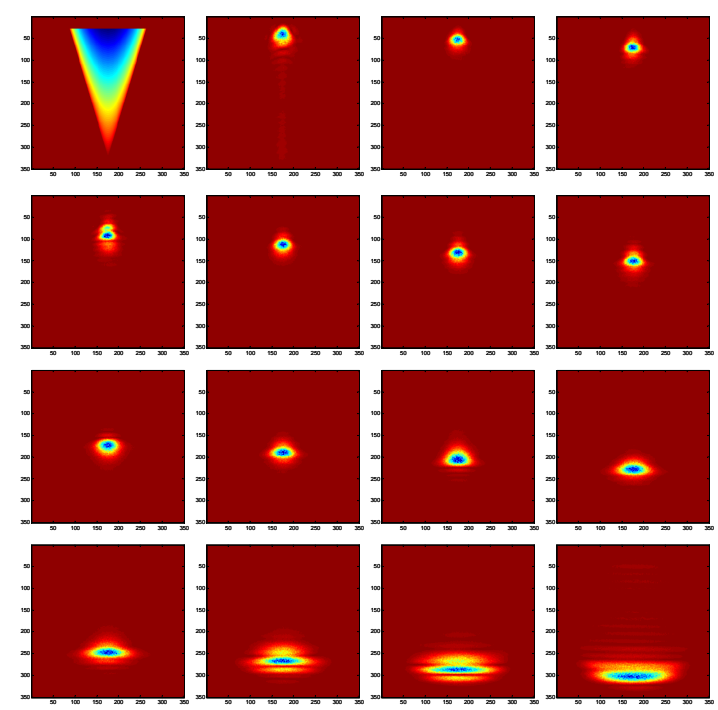

Figure 4.1: Returning $x$-polarized fields at receiver plane for R-R scattering model, where $\sigma_{s} / \lambda=.02$ and $l_{c} / \lambda=2$.

$$
\Omega=\frac{A_{d}}{z^{2}}
$$

where $A_{d}$ is the area of the collecting aperture and $z$ is the distance from the target to the collecting aperture. In turn, the scattered irradiance is converted to the optical field using equation 2.76. The optical field is then propagated back through the atmosphere, to the aperture plane. Figures 4.1, 4.2, 4.3, 4.4, 4.5 and 4.6 show the absolute value of the returning optical field at the aperture plane for various targets. Figures 4.7 and 4.8 show the total energy contained in the series of return pulses as a function of time and $\sigma_{s} / \lambda$ for a downward pointing conical target. Figures 4.11 and 4.12 show the total energy contained in the series of return pulses as a function of time and $l_{c} / \lambda$ for the same conical target. Figures 4.9 and 4.10 show the total energy contained in the series of return pulses as a function of time and $\sigma_{s} / \lambda$ for a conical target set at an angle to the vertical. Figures 4.13 and 4.14 show the total energy contained in the series of return pulses as a function of time and $l_{c} / \lambda$ for the same angled conical target. The energy shown in these graphs is the average energy over 200 different atmospheric iterations.

\subsection{Backscattering from Beckmann-Kirchhoff Surface}

Unlike the Rayleigh-Rice model, the Beckmann-Kirchhoff model requires the incident irradiance (equation 2.76) which is multiplied by equation 3.20 to give scattered radiance. 


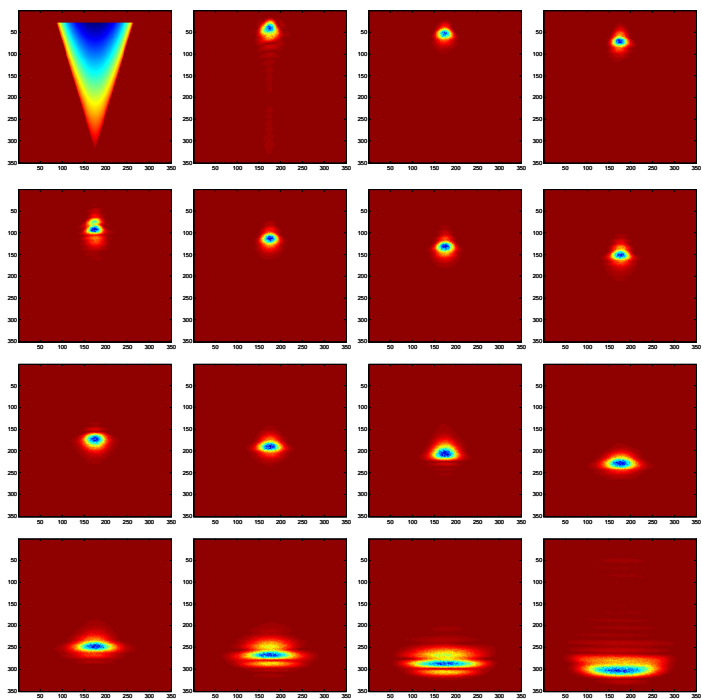

Figure 4.2: Returning $y$-polarized fields at receiver plane for R-R scattering model, where $\sigma_{s} / \lambda=.02$ and $l_{c} / \lambda=2$.

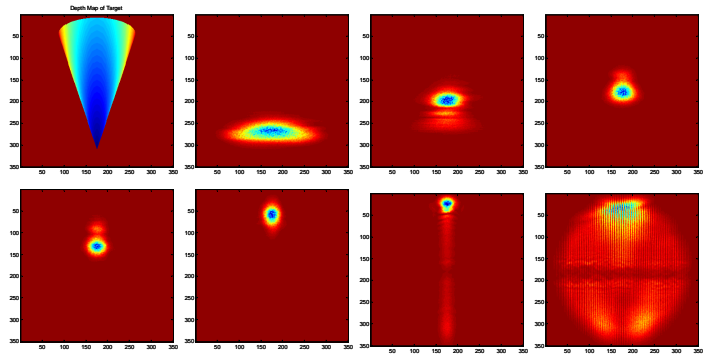

Figure 4.3: Returning $x$-polarized fields at receiver plane for R-R scattering model, where $\sigma_{s} / \lambda=.02$ and $l_{c} / \lambda=2$.

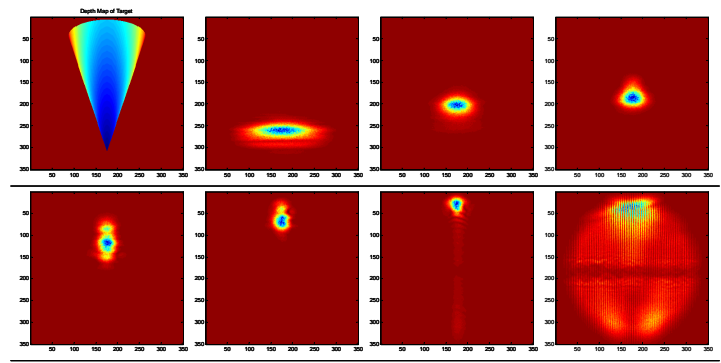

Figure 4.4: Returning $y$-polarized fields at receiver plane for R-R scattering model, where $\sigma_{s} / \lambda=.02$ and $l_{c} / \lambda=2$. 


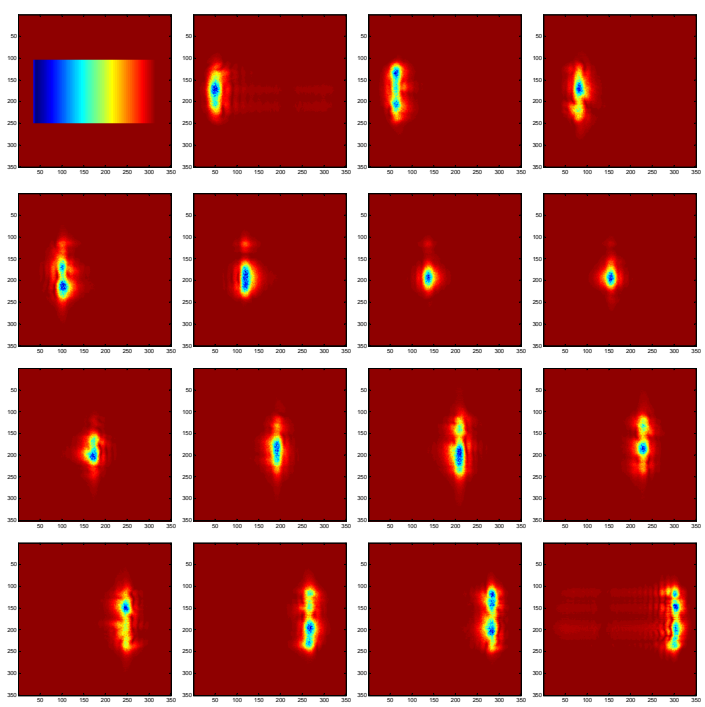

Figure 4.5: Returning $x$-polarized fields at receiver plane for R-R scattering model, where $\sigma_{s} / \lambda=.02$ and $l_{c} / \lambda=2$.
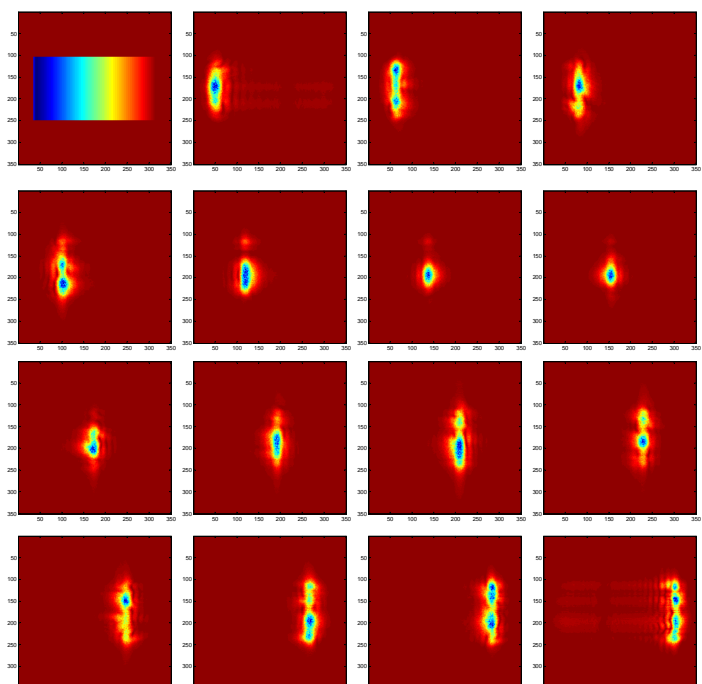

Figure 4.6: Returning $y$-polarized fields at receiver plane for R-R scattering model, where $\sigma_{s} / \lambda=.02$ and $l_{c} / \lambda=2$. 

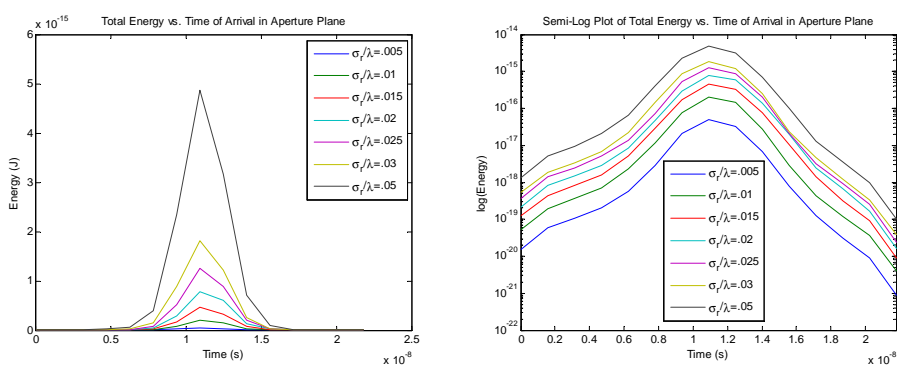

Figure 4.7: Energy of $x$-polarized pulse in aperture plane as a function of time of arrival and surface roughness for the R-R scattering model. The target is the downward pointing cone. $l_{c} / \lambda$ is held constant at 2 .
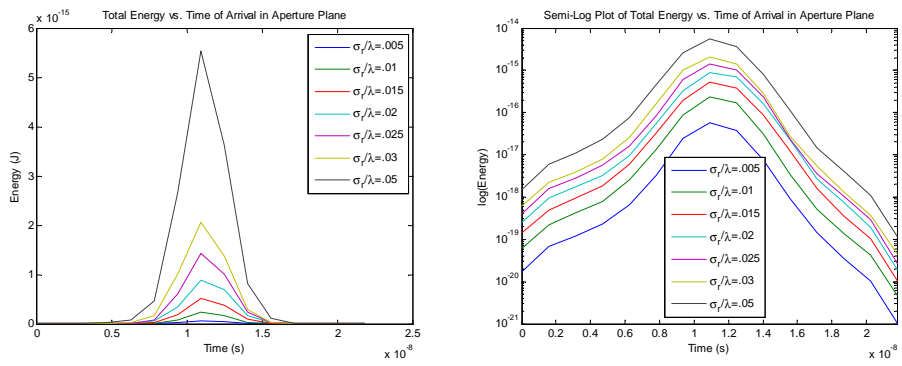

Figure 4.8: Energy of $y$-polarized pulse in aperture plane as a function of time of arrival and surface roughness for the R-R scattering model. The target is the downward pointing cone. $l_{c} / \lambda$ is held constant at 2 .
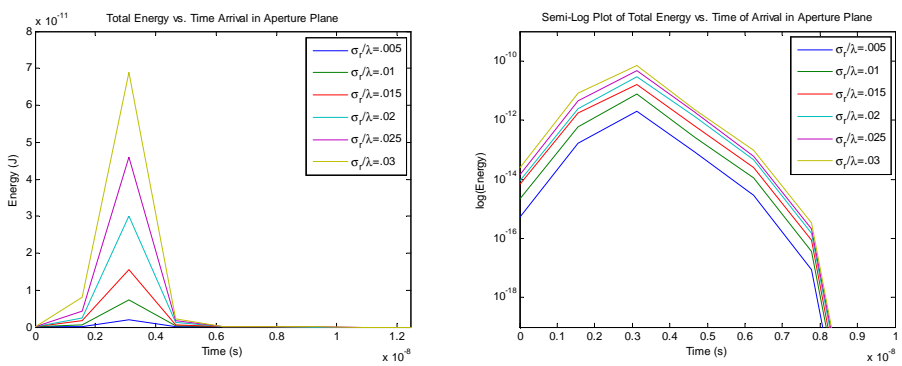

Figure 4.9: Energy of $x$-polarized pulse in aperture plane as a function of time of arrival and surface roughness for the R-R scattering model. The target is the angled cone. $l_{c} / \lambda$ is held constant at 2 . 

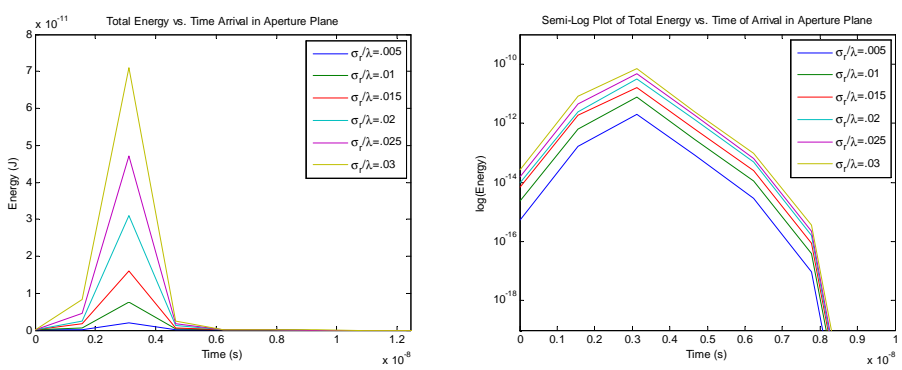

Figure 4.10: Energy of $y$-polarized pulse in aperture plane as a function of time of arrival and surface roughness for the R-R scattering model. The target is the angled cone. $l_{c} / \lambda$ is held constant at 2 .
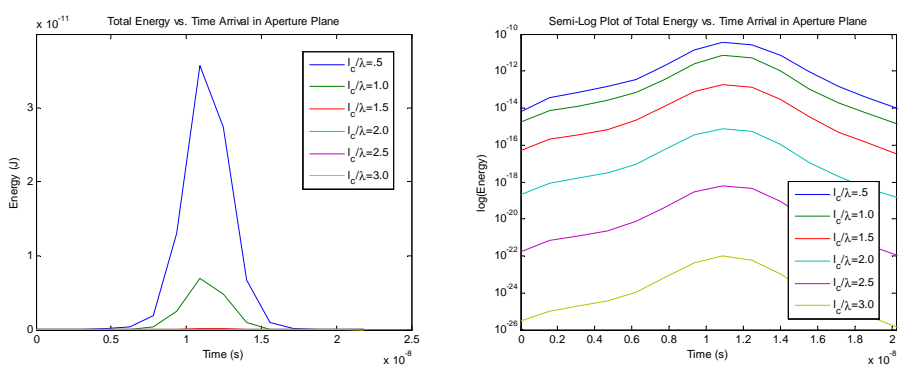

Figure 4.11: Energy of $x$-polarized pulse in aperture plane as a function of time of arrival and autocovariance length for the R-R scattering model. The target is the downward pointing cone. $\sigma_{s} / \lambda$ is held constant at .02 .
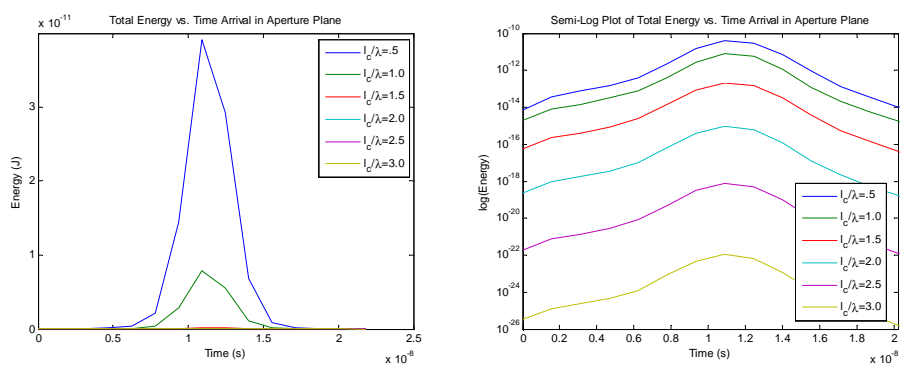

Figure 4.12: Energy of $y$-polarized pulse in aperture plane as a function of time of arrival and autocovariance length for the R-R scattering model. The target is the downward pointing cone. $\sigma_{s} / \lambda$ is held constant at .02 . 

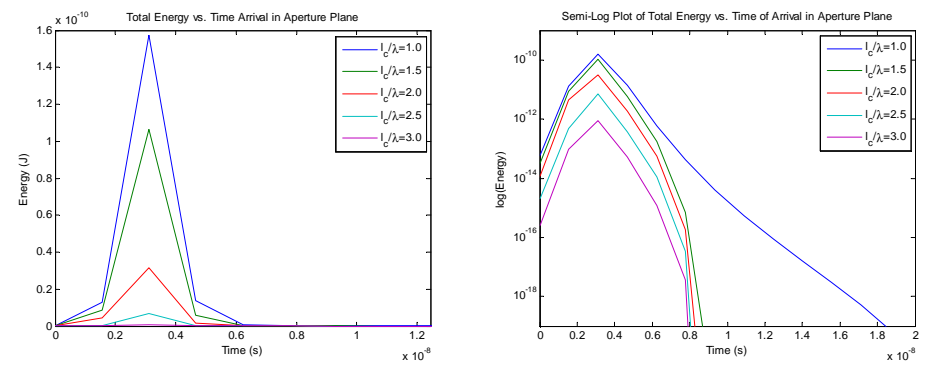

Figure 4.13: Energy of $x$-polarized pulse in aperture plane as a function of time of arrival and autocovariance length for the R-R scattering model. The target is the angled cone. $\sigma_{s} / \lambda$ is held constant at .02 .
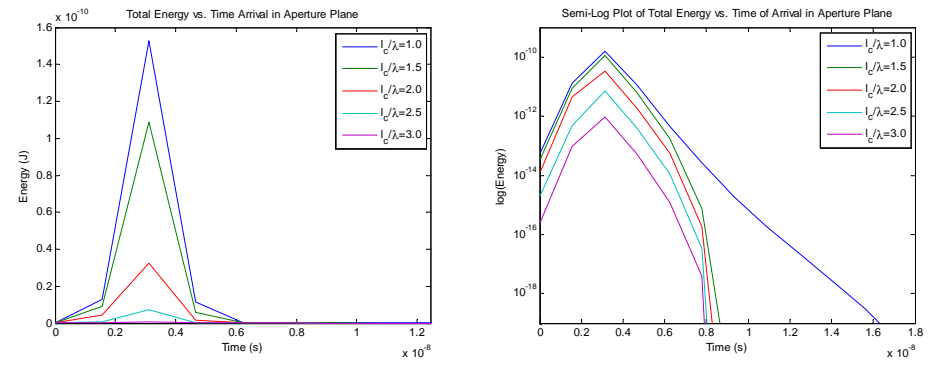

Figure 4.14: Energy of $y$-polarized pulse in aperture plane as a function of time of arrival and autocovariance length for the R-R scattering model. The target is the angled cone. $\sigma_{s} / \lambda$ is held constant at .02 . 

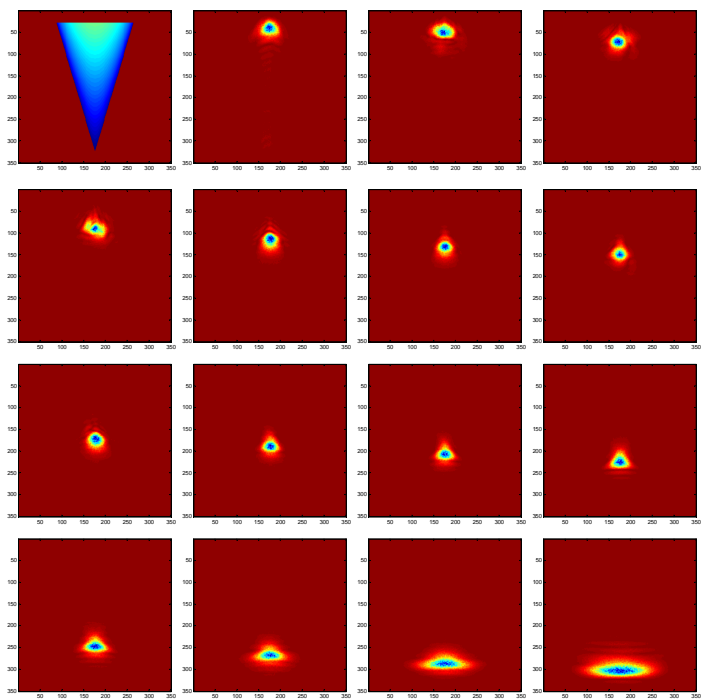

Figure 4.15: Returning fields at receiver plane for B-K scattering model, where $\sigma_{s} / \lambda=.1$ and $l_{c} / \lambda=2$.

The scattered radiance is converted to scattered irradiance using [13]

$$
E_{S}(x, y)=L_{s}(x, y) \Omega
$$

where $\Omega$ is given by equation 4.2 above. Recall that the B-K scattering model is a scalar model and does not involve polarization effects. The scattered irradiance from equation 4.3 is converted to the optical field using equation 2.76. The optical field is then propagated back through the atmosphere, to the aperture plane. Figures 4.15, 4.16 and 4.17 show the absolute value of the returning optical field at the aperture plane. Figure 4.18 shows the total energy contained in the series of return pulses as a function of time and $l_{c} / \lambda$ for the same conical target. Figure 4.19 shows the total energy contained in the series of return pulses as a function of time and $\sigma_{s} / \lambda$ for a downward pointing conical target. The energy shown in these graphs is the average energy over 200 different atmospheric iterations. 


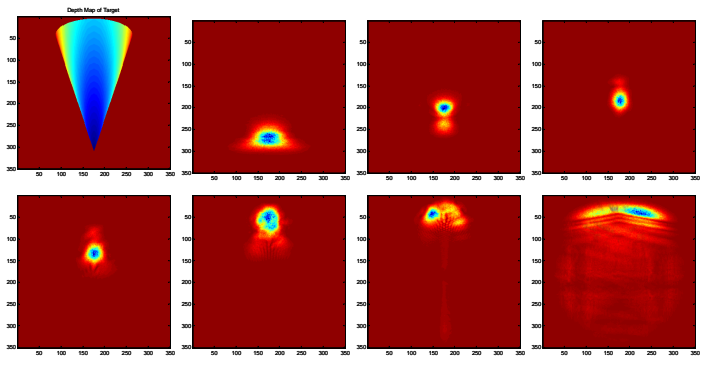

Figure 4.16: Returning fields at receiver plane for B-K scattering model, where $\sigma_{s} / \lambda=.5$ and $l_{c} / \lambda=2$.

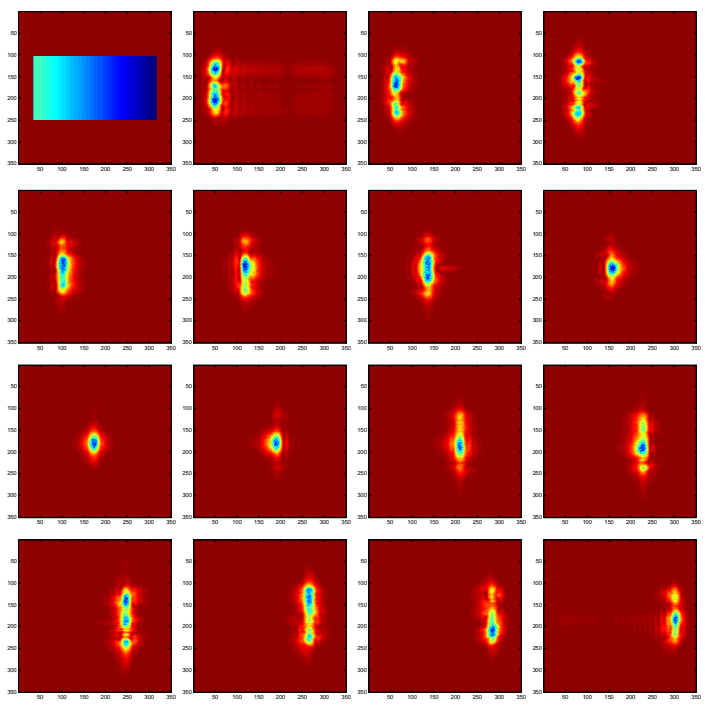

Figure 4.17: Returning fields at receiver plane for B-K scattering model, where $\sigma_{s} / \lambda=.1$ and $l_{c} / \lambda=2$. 

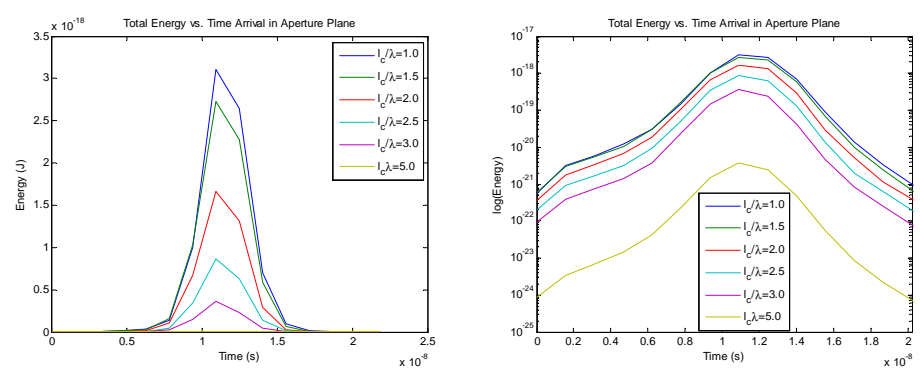

Figure 4.18: Energy of pulse in aperture plane as a function of time of arrival and autocovariance length for the B-K scattering model. The target is the downward pointing cone. $\sigma_{S} / \lambda$ is held constant at .25 .
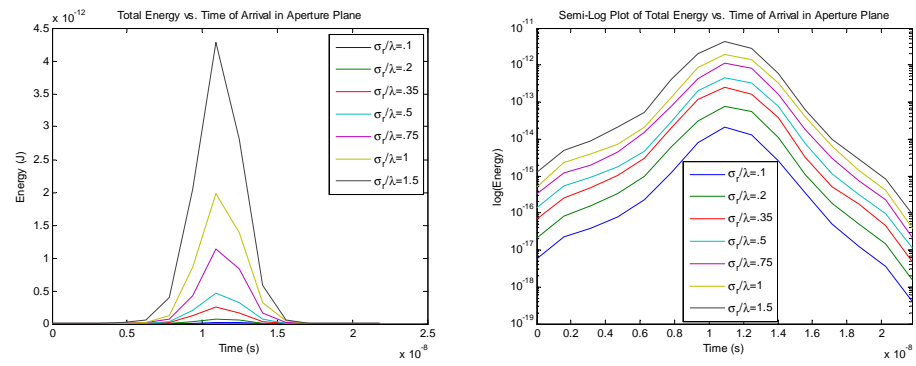

Figure 4.19: Energy of pulse in aperture plane as a function of time of arrival and surface roughness for the B-K scattering model. The target is the downward pointing cone. $l_{c} / \lambda$ is held constant at 1 .

\subsection{Backscattering from Microfacet Surface}

The BRDF of the microfacet model also requires the incident irradiance, and returns the scattered irradiance. Equation 4.3 is used to convert scattered radiance to scattered irradiance. The scattered irradiance is converted to the optical field using equation 2.76. The optical field is then propagated back through the atmosphere, to the aperture plane. Figures $4.20,4.21,4.22$ and 4.23 show the absolute value of the returning optical field at the aperture plane. 


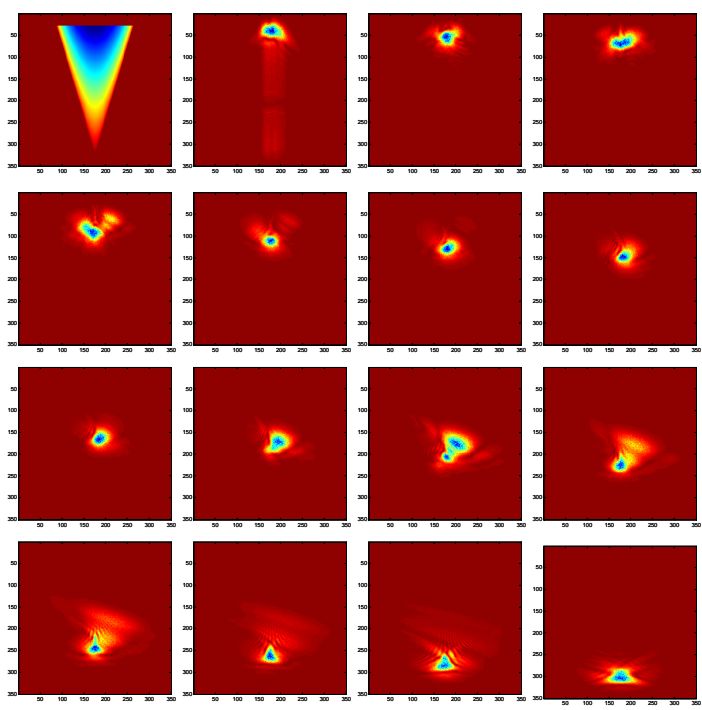

Figure 4.20: Returning $x$-polarized fields at receiver plane for MF scattering model.

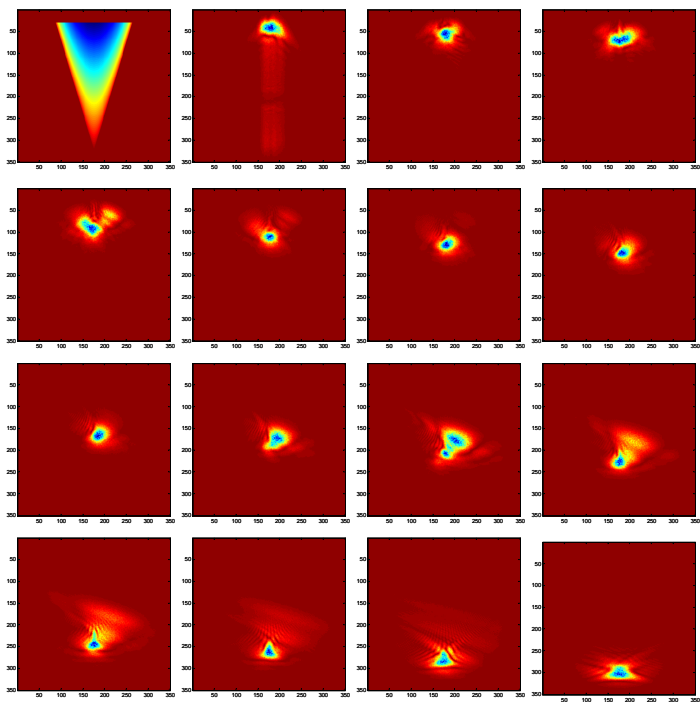

Figure 4.21: Returning y-polarized fields at receiver plane for MF scattering model. 

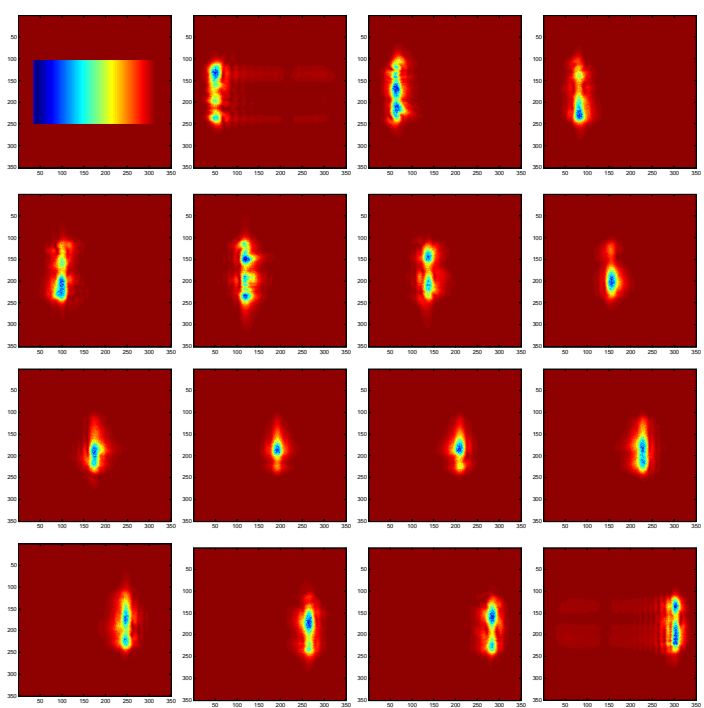

Figure 4.22: Returning $x$-polarized fields at receiver plane for MF scattering model.
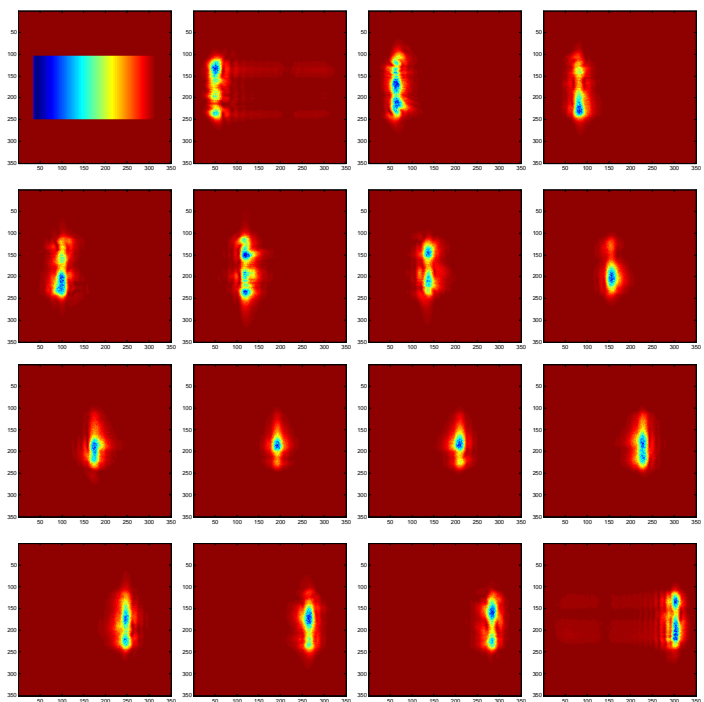

Figure 4.23: Returning $y$-polarized fields at receiver plane for MF scattering model. 


\section{Chapter 5}

\section{Conclusion}

\subsection{Discussion of Results}

The goal of this simulation was to determine if the PPR concept can be used to determine spatial and material information of a target object in orbit. By viewing the returning optical field from each slice, we can get a view of the shape and orientation of the target. The return pulse for a particular target slice is centered on the same location in the array where the incident pulse encountered the slice. If nothing else, this information can tell us if the surface boundary of the object exists in a certain depth plane. By evaluating the shape of the return pulse, we can extract more information. The simplest example of this is for the rectangular prism target. Figures 4.5, 4.6, 4.17, 4.22 and 4.23 all show results from the rectangular prism with an incident angle of $\theta_{i}=20^{\circ}$. The incoming pulse first strikes the left edge, and continues along the body of the prism and last strikes the right edge. At each slice, the pulse encounters a rectangle which can be $\operatorname{describe}$ as $\operatorname{rect}\left(h_{x} x\right) \operatorname{rect}\left(h_{y} y\right)$ where $h_{x}$ and $h_{y}$ give the length of the rectangle in the $x$ and $y$ directions and [1]

$$
\operatorname{rect}(x)=\left\{\begin{array}{cc}
1 & |x|<\frac{1}{2} \\
\frac{1}{2} & |x|=\frac{1}{2} \\
0 & \text { otherwise }
\end{array} .\right.
$$

Regardless of the scattering model each of the return pulses exhibit similar characteristics to the two dimensional sinc function which is the Fourier transform pair of the two dimensional rect function. I believe that this information could be used to recreate the target's shape and orientation using the optical field at the receiver. Figure 5.1 shows a slice across the center of an image scattered from a R-R object which exhibits the typical sinc function lobes.

Variations in surface roughness and autocovariance length change the average total pulse energy as shown in figures 4.7, 4.8, 4.11, 4.12, 4.19 and 4.18. As the surface rough- 


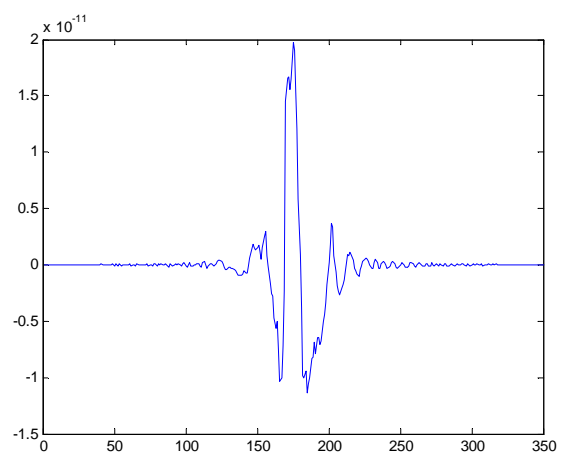

Figure 5.1: Horizontal slice across center of return pulse exhibiting sinc function behavior.

ness is increased, the radiation will be scattered over a greater angle. This means less energy will be scattered in the direction of the receiver. As the autocovariance length is increased, the radiation will be scattered over a tighter angle. This means more energy will be scattered in the direction of the receiver. This behavior was also shown in the scattering distributions at the end of sections 3.2 and 3.3. I believe the best way to use this information is to create a look-up table. Simulations would be run for various values of surface roughness, autocovariance length and dielectric constant to create the look-up table. Trials could then be run with unknown surface characteristics and compared to the look-up table to determine specific surface characteristics.

\subsection{Areas for Future Work}

The most interesting area for future work would be recreation of the target's shape and orientation using the incoming optical field. I think this could be done by propagation each time-slice of the incoming pulse back to the target plane. By positioning these reconstructions in the correct positions, based on time on arrival, I believe the a model target could be formed. Some type of averaging would have to be preformed to adjust for the effects of the atmosphere. By adjusting the time resolution of the system, more return pulses could be made. This would increase the accuracy of the spatial information collected. This does come at the cost of higher memory requirements, however. I ran into memory trouble in MatLab when running more than 12 atmospheric iterations at a time. To run with a higher time resolution, fewer atmospheric iterations would be preformed. 


\section{Bibliography}

[1] J.W. Goodman, Introduction to Fourier Optics, 3rd ed. Englewood,CO: Roberts \& Company, 2005.

[2] E. Hecht, Optics, 4th ed. San Fransisco: Pearson Education, 2002.

[3] A. Krywonos, Predicting Surface Scatter Using a Linear Systems Formulation of Non-paraxial Scalar Diffraction.

[4] J.C. Stover, Optical Scattering, Measurement and Analysis. New York: McGraw-Hill, 1990.

[5] J.W. Goodman, Statistical Optics. New York: Wiley-Interscience, 1985.

[6] H. Hemmati, Deep Space Optical Communication. Hoboken, NJ: Wiley-Interscience, 2006.

[7] M.C. Roggemann and B.M. Walsh, Imaging Through Turbulence. Boca Raton, FL: CRC Press, 1996.

[8] J.E. Harvey, "Modeling the image quality of enhanced reflectance x-ray multilayers as a surface power spectral density filter function", Applied Optics 34, pp. 3715-3726, 1995.

[9] E.W. Weisstein, Hanning Function, From Mathworld-A Wolfram Web Resource. http://mathworld.wolfram.com/HanningFunction.html

[10] C. Huygens, Treatise on Light, New York: Dover, 1962.

[11] M. Nieto-Vesperinas, Scattering and Diffraction in Physical Optics. New York: Wiley-Interscience, 1991.

[12] A. Papoulis and S.U. Pillai, Probability, Random Variables and Stochastic Processes, 4th ed. Boston: McGraw-Hill, 2002. 
[13] R.W. Boyd, Radiometry and the Detection of Optical Radiation. New York: John Wiley and Sons, 1983.

[14] R.G. Lane, A. Glindemann and J.C. Dainty, "Simulation of a Kolmogorov phase screen", Waves in Random and Complex Media, pp 209-224, 1992.

[15] J. Stryjewski, Microfacet Scattering Model for PPR

[16] M. Ashikhmin, S. Premose, P. Shirley, “A Microfacet-based BRDF Generator”, SIGGRAPH, pp 65-74, 2000.

[17] I. Newton, Opticks. New York: Dover, 1952.

[18] R. Hooke, Micrographia. New York: Dover, 1961.

[19] T. Young, Miscellaneous Works of the Late Thomas Young. London: John Murray, 1855.

[20] F.M. Grimaldi, De Lumine. London: Dawsons of Pall Mall, 1966.

[21] J.L. Vaughn, PHZSCRN.m, MatLab code. Anaheim, CA: the Optical Sciences Company, 2001. 